\title{
Mean-field optimal control as Gamma-limit of finite agent controls
}

\author{
Massimo Fornasier * $\quad$ Stefano Lisini ${ }^{\dagger} \quad$ Carlo Orrieri ${ }^{\ddagger} \quad$ Giuseppe Savaré ${ }^{\S}$
}

March 14, 2018

\begin{abstract}
This paper focuses on the role of a government of a large population of interacting agents as a mean field optimal control problem derived from deterministic finite agent dynamics. The control problems are constrained by a PDE of continuity-type without diffusion, governing the dynamics of the probability distribution of the agent population. We derive existence of optimal controls in a measure-theoretical setting as natural limits of finite agent optimal controls without any assumption on the regularity of control competitors. In particular, we prove the consistency of mean-field optimal controls with corresponding underlying finite agent ones. The results follow from a $\Gamma$-convergence argument constructed over the mean-field limit, which stems from leveraging the superposition principle.
\end{abstract}

Keywords: finite agent optimal control, mean-field optimal control, $\Gamma$-convergence, superposition principle

\section{Introduction}

In the mathematical modelling of biological, social, and economical phenomena, self-organization of multi-agent interaction systems has become a focus of applied mathematics and physics and mechanisms are studied towards the formation of global patterns. In the last years there has been a vigorous development of literature describing collective behaviour of interacting agents 29 31, 40 42, 60, towards modeling phenomena in biology, such as motility and cell aggregation [15, 43, 44, 53, coordinated animal motion [7, 20, 23, 25, 27, 31, 49, 51, 55, 59, 63, coordinated human [28, 33, 57 and synthetic agent interactions and behaviour, as in the case of cooperative robots [24, 48, 52, 58. Part of the literature is particularly focused on studying corresponding mean-field equations in order to simplify models for large populations of interacting agents: the effect of all the other individuals on any given individual is described by a single averaged effect. As it is very hard to be exhaustive in accounting all the developments of this very fast growing field, we refer to $18,19,21,22,61$, for recent surveys.

Self-organization is an incomplete concept, see, e.g. [12], as it is not always occurring when needed. In fact, local interactions between agents can be interpreted as distributed controls, which however are not always able to lead to global coordination or pattern formation. This motivated the research also of centralized optimal controls for multi-agent systems, modeling the intervention of

\footnotetext{
*Department of Mathematics, TU München. Boltzmannstr. 3, Garching bei München, D-85748, Germany. massimo.fornasier@ma.tum.de

†Dipartimento di Matematica "F. Casorati", Università di Pavia. Via Ferrata 5, 27100 Pavia, Italy. stefano.lisini@unipv.it

‡Dipartimento di Matematica "G. Castelnuovo", Sapienza Università di Roma. Piazzale Aldo Moro 5, 00185 Roma, Italy. orrieri@mat. uniroma1.it

§Dipartimento di Matematica "F. Casorati", Università di Pavia. Via Ferrata 5, 27100 Pavia, Italy. giuseppe.savare@unipv.it
} 
an external government to induce desired dynamics or pattern formation. In this paper we are concerned with the control of deterministic multi-agent systems of the type

$$
\dot{x}_{i}(t)=\boldsymbol{F}^{N}\left(x_{i}(t), \boldsymbol{x}(t)\right)+u_{i}(t), \quad i=1, \cdots, N .
$$

The map $\boldsymbol{F}^{N}: \mathbb{R}^{d} \times\left(\mathbb{R}^{d}\right)^{N} \rightarrow \mathbb{R}^{d}$ models the interaction between the agents and $\boldsymbol{u}$ represents the action of an external controller on the system. The control is optimized by minimization of a cost functional

$$
\mathcal{E}^{N}(\boldsymbol{x}, \boldsymbol{u}):=f_{0}^{T} \frac{1}{N} \sum_{i=1}^{N} L^{N}\left(x_{i}(t), \boldsymbol{x}(t)\right) \mathrm{d} t+f_{0}^{T} \frac{1}{N} \sum_{i=1}^{N} \psi\left(u_{i}(t)\right) \mathrm{d} t,
$$

where $L^{N}$ is a suitable cost function used for modelling the goal of the control and capturing the work done to achieve it, and $\psi$ is an appropriate positive convex function, which is superlinear at infinity and models the effective cost of employing the control. When the number $N$ of agents is very large, dynamical programming for solving the optimal control problem defined by minimization of (1.2) under the constraints (1.1) become computationally intractable. In fact, Richard Bellman coined the term "curse of dimensionality" precisely to describe this phenomenon.

For situations where agents are indistinguishable, e.g. drawn independently at random from an initial probability distribution $\mu_{0}$, and the dynamics $\boldsymbol{F}^{N}\left(x_{i}(t), \boldsymbol{x}(t)\right)=\boldsymbol{F}^{N}\left(x_{i}(t), \mu_{t}^{N}\right)$ depends in fact from the empirical distribution $\mu_{t}^{N}=\frac{1}{N} \sum_{i=1}^{N} \delta_{x_{i}(t)}$ one may hope to invoke again the use of mean-field approximations for a tractable (approximate) solution of the control problem. By formally considering the mean-field limit of the system (1.1) for $N \rightarrow \infty$ one obtains the continuity equation of Vlasov-type

$$
\partial_{t} \mu_{t}+\nabla \cdot\left(\left(\boldsymbol{F}\left(x, \mu_{t}\right)+\boldsymbol{v}_{t}\right) \mu_{t}\right)=0 \quad \text { in }(0, T) \times \mathbb{R}^{d},
$$

where $\mu$ is the weak limit of $\mu^{N}$ and represents the (time dependent) probability distribution of agents, and $\boldsymbol{\nu}=\boldsymbol{v} \mu$ is a suitable vector control measure absolutely continuous w.r.t. $\mu$ and subjected to a cost functional

$$
\mathcal{E}(\mu, \boldsymbol{\nu}):=f_{0}^{T} \int_{\mathbb{R}^{d}} L\left(x, \mu_{t}\right) \mathrm{d} \mu_{t}(x) \mathrm{d} t+f_{0}^{T} \int_{\mathbb{R}^{d}} \psi(\boldsymbol{v}(t, x)) \mathrm{d} \mu_{t}(x) \mathrm{d} t,
$$

The vector measure $\boldsymbol{\nu}=\boldsymbol{v} \mu$ can in fact be obtained as the weak limit of the sequence of finite dimensional control measures

$$
\boldsymbol{\nu}^{N}=f_{0}^{T} \delta_{t} \otimes \boldsymbol{\nu}_{t}^{N} \mathrm{~d} t, \quad \boldsymbol{\nu}_{t}^{N}=\frac{1}{N} \sum_{i=1}^{N} u_{i}(t) \delta_{x_{i}(t)}, \quad t \in[0, T] .
$$

Under suitable assumptions on $\psi$, on the convergence of $\boldsymbol{F}^{N}$ to $\boldsymbol{F}$ and of $L^{N}$ to $L$, and assuming for simplicity that the initial data are confined in a compact subset of $\mathbb{R}^{d}$, one of the main result of this paper can be summarized as follows.

Theorem 1.1 If the initial measures $\mu_{0}^{N}=\frac{1}{N} \sum_{i=1}^{N} \delta_{x_{i}^{N}(0)}$ weakly converge to a limit probability measure $\mu_{0}$ then the minimum $E^{N}\left(\mu_{0}^{N}\right)$ of (1.2) among all the solution of the controlled system (1.1) converges to the minimum $E\left(\mu_{0}\right)$ of the functional (1.4) among all the solutions of (1.3) with initial datum $\mu_{0}$. Moreover, all the accumulation points (in the topology of weak convergence of measures) of the measures associated to minimizers $\boldsymbol{x}^{N}, \boldsymbol{u}^{N}$ of (1.2) are minima of (1.4).

The idea of solving finite agent optimal control problems by considering a mean-field approximation has been considered since the 1960's [36,37,45] with the introduction of stochastic optimal control. The optimal control of stochastic differential equations

$$
d X_{t}^{i}=\boldsymbol{F}\left(X_{t}^{i}, \mu_{t}^{N}\right)+\boldsymbol{v}\left(t, X_{t}^{i}\right)+\sigma d W_{t}^{i}, \quad i=1, \cdots, N ;
$$


with non-degenerate diffusion and independent Brownian motions $W^{i}$, has been for a long while studied via the optimal control of the law $\mu_{t}=\operatorname{Law}\left(X_{t}\right)$ constrained by a McKean-Vlasov equation

$$
\partial_{t} \mu_{t}+\nabla \cdot\left(\left(\boldsymbol{F}\left(x, \mu_{t}\right)+\boldsymbol{v}(t, x)\right) \mu_{t}\right)=\sigma \Delta \mu_{t},
$$

under a suitable control cost

$$
\mathcal{E}(\mu, \boldsymbol{v}):=f_{0}^{T} \int_{\mathbb{R}^{d}} L\left(x, \mu_{t}\right) \mathrm{d} \mu_{t}(x) \mathrm{d} t+f_{0}^{T} \int_{\mathbb{R}^{d}} \psi(\boldsymbol{v}(t, x)) \mathrm{d} \mu_{t}(x) \mathrm{d} t .
$$

Most of the literature on stochastic control is focused primarily on the solution of McKean-Vlasov optimal control problems. The most popular methods are based on extending Pontryagin's maximum principle [2, 6, 9, 14, 17, or deriving a dynamic programming principle, and with it a form of a Hamilton-Jacobi-Bellman equation on a space of probability measures [8, 47, 54. However, the rigorous justification that the McKean-Vlasov optimal control problem is consistent with the limit of optimal controls for stochastic finite agent models has been proved surprisingly just very recently [46]. The techniques used in the latter paper are largely based on martingale problems, combining ideas from the McKean-Vlasov limit theory with a well-established compactification method for stochastic control 36 .

Due to the probabilistic nature of the methods used for stochastic control, the consistency results become weaker for the limiting case of vanishing viscosity $\sigma \rightarrow 0$ as in (1.3) (see e.g. 46)). Sharp results for purely deterministic dynamics (1.1) require indeed measure-theoretical methods. The first work addressing the consistency of mean-field optimal control for deterministic finite agent systems is 39. In the latter paper an analogous result as Theorem 1.1 is derived for general penalty functions $\psi$ with polynomial growth, including the interesting case of linear growth at 0 and infinity, motivated by results of sparse controllability for finite-agent models [10,11,16. Other models of sparse mean-field optimal control have been considered in [1,13, 38. The generality of the penalty function $\psi$ in [39] has required to restrict the class of controls: they have been assumed to be locally Lipschitz continuous in space feedback control functions $u_{i}(t)=\boldsymbol{v}\left(t, x_{i}\right)$ with controlled time-dependent Lipschitz constants.

In this paper and in our main result Theorem 1.1 we remove this restriction, but we still impose suitable coercivity on the admissible controls, by requiring the function $\psi$ to have superlinear growth to infinity. As sparsity of controls, i.e. the localization of controls in space, is mainly due to the linear behaviour of the penalty function at 0 , the superlinear growth at infinity does not exclude the possibility of using this model for sparse control. Moreover, in this framework there is no need for enforcing a priori that controls are smooth feedback functions of the state variables and the limit process comes very natural in a measure-threoretical sense. In view of the minimal smoothness required to the governing interaction functions $\boldsymbol{F}^{N}, \boldsymbol{F}$ (they are assumed to be just continuous) there is no uniqueness of solutions in general of (1.1) and (1.3). Hence, the main results of mean-field limit are derived by leveraging the powerful machinery of the superposition principle [3, Section 3.4].

The paper is organized as follows: after recalling in details the notation and a few preliminary results on optimal transport, doubling functions and convex functionals on measures in Section 2, we describe our setting of optimal control problems in Section 3, together with the precise statements of our main results. We address the existence of solutions of the finite agent optimal control problem in Section 4. Crucial moment estimates are derived in Section 5 for feasible competitors for the mean-field control problem, which are useful for deriving compactness arguments further below. Section 6 is dedicated to the proofs of our main Theorems. A relevant part is devoted to Theorem 1.1 by developing a $\Gamma$-convergence argument. While the $\Gamma-\lim$ inf inequality follows by relatively standard lower-semicontinuity arguments, the derivation of the $\Gamma-\lim$ sup inequality requires a technical application of the superposition principle. Equi-coercivity and convergence of minimizers follow from compactness arguments based on moment estimates from Section 5 . 


\section{Notation and preliminary results}

Throughout the paper we work with $\mathbb{R}^{d}$ as a state space and we fix a time horizon $T>0$. We will denote by $\lambda$ the normalized restriction of the Lebesgue measure to $[0, T], \lambda:=\frac{1}{T} \mathscr{L}^{1}\llcorner[0, T]$.

Given $(\mathcal{S}, d)$ a metric space, we use the classical notation $A C([0, T] ; \mathcal{S})$ for the classes of $\mathcal{S}$-valued absolute continuous curves. We indicate with $\mathcal{M}\left(\mathbb{R}^{d}\right), \mathcal{M}\left(\mathbb{R}^{d} ; \mathbb{R}^{d}\right)$ the space of Borel (vector-valued) measures.

\subsection{Probability measures and optimal transport costs}

We call $\mathcal{P}\left(\mathbb{R}^{d}\right)$ the space of Borel probability measures. If $f: \Omega \rightarrow \mathbb{R}^{h}$ is a Borel map defined in a Borel subset $\Omega$ of $\mathbb{R}^{d}$, and $\mu \in \mathcal{P}\left(\mathbb{R}^{d}\right)$ is concentrated on $\Omega$, we will denote by $f_{\sharp} \mu$ the Borel measure in $\mathbb{R}^{h}$ defined by $f_{\sharp} \mu(B):=\mu\left(f^{-1}(B)\right)$, for every Borel subset $B \subset \mathbb{R}^{h}$.

Whenever $\psi: \mathbb{R}^{d} \rightarrow[0,+\infty]$ is a lower semicontinuous function, we set

$$
\mathcal{C}_{\psi}\left(\mu_{0}, \mu_{1}\right):=\inf \left\{\int_{\mathbb{R}^{d} \times \mathbb{R}^{d}} \psi(y-x) \mathrm{d} \gamma(x, y): \gamma \in \Pi\left(\mu_{0}, \mu_{1}\right)\right\},
$$

where $\Pi\left(\mu_{0}, \mu_{1}\right)$ is the set of the optimal transport plans:

$$
\Pi\left(\mu_{0}, \mu_{1}\right):=\left\{\gamma \in \mathcal{P}\left(\mathbb{R}^{d} \times \mathbb{R}^{d}\right): \gamma\left(B \times \mathbb{R}^{d}\right)=\mu_{0}(B), \gamma\left(\mathbb{R}^{d} \times B\right)=\mu_{1}(B) \quad \forall B \text { Borel set in } \mathbb{R}^{d}\right\} .
$$

In the particular case when $\psi(z):=|z|, z \in \mathbb{R}^{d}$, (2.1) defines the $L^{1}$-Wasserstein distance

$$
W_{1}\left(\mu_{0}, \mu_{1}\right):=\inf \left\{\int_{\mathbb{R}^{d} \times \mathbb{R}^{d}}|x-y| \mathrm{d} \gamma(x, y): \gamma \in \Pi\left(\mu_{0}, \mu_{1}\right)\right\} ;
$$

the infimum in (2.2) is always finite and attained if $\mu_{0}, \mu_{1}$ belong to the space $\mathcal{P}_{1}\left(\mathbb{R}^{d}\right)$ of Borel probability measure with finite first order moment:

$$
\mathcal{P}_{1}\left(\mathbb{R}^{d}\right):=\left\{\mu \in \mathcal{P}\left(\mathbb{R}^{d}\right): \int_{\mathbb{R}^{d}}|x| \mathrm{d} \mu(x)<+\infty\right\} .
$$

$\mathcal{P}_{1}\left(\mathbb{R}^{d}\right)$ endowed with $W_{1}\left(\mu_{0}, \mu_{1}\right)$ is a complete and separable metric space. In particular we will consider absolutely continuous curves $t \mapsto \mu_{t}$ in $A C\left([0, T] ; \mathcal{P}_{1}\left(\mathbb{R}^{d}\right)\right)$. They will canonically induce a parametrized measure $\tilde{\mu}:=\int \delta_{t} \otimes \mu_{t} \mathrm{~d} \lambda(t)$ in $\mathcal{P}_{1}\left([0, T] \times \mathbb{R}^{d}\right)$, satisfying

$$
\int f(t, x) \mathrm{d} \tilde{\mu}(t, x)=\int_{0}^{T} \int_{\mathbb{R}^{d}} f(t, x) \mathrm{d} \mu_{t}(x) \mathrm{d} \lambda(t)=f_{0}^{T} \int_{\mathbb{R}^{d}} f(t, x) \mathrm{d} \mu_{t}(x) \mathrm{d} t .
$$

Convergence with respect to $W_{1}$ is equivalent to weak convergence (in duality with continuous and bounded functions) supplemented with convergence of first moment; equivalently, for every sequence $\left(\mu_{n}\right)_{n \in \mathbb{N}} \subset \mathcal{P}_{1}\left(\mathbb{R}^{d}\right)$ and candidate limit $\mu \in \mathcal{P}_{1}\left(\mathbb{R}^{d}\right)$

$$
\lim _{n \rightarrow \infty} W_{1}\left(\mu_{n}, \mu\right)=0 \Leftrightarrow \lim _{n \rightarrow \infty} \int \zeta \mathrm{d} \mu_{n}=\int \zeta \mathrm{d} \mu \quad \text { for every } \zeta \in C\left(\mathbb{R}^{d}\right), \sup _{x \in \mathbb{R}^{d}} \frac{\zeta(x)}{1+|x|}<\infty .
$$

In $\mathcal{P}_{1}\left(\mathbb{R}^{d}\right)$ we consider the subset $\mathcal{P}^{N}\left(\mathbb{R}^{d}\right)$ of discrete measures

$$
\mathcal{P}^{N}\left(\mathbb{R}^{d}\right):=\left\{\mu=\frac{1}{N} \sum_{i=1}^{N} \delta_{x_{i}} \text { for some } x_{i} \in \mathbb{R}^{d}\right\} .
$$

A measure $\mu$ belongs to $\mathcal{P}^{N}\left(\mathbb{R}^{d}\right)$ if and only if $\# \operatorname{supp}(\mu) \leq N$ and $N \mu(B) \in \mathbb{N}$ for every Borel set $B$ of $\mathbb{R}^{d}$. Let us now fix an integer $N \in \mathbb{N}$ and consider vectors $\boldsymbol{x}=\left(x_{1}, \cdots, x_{N}\right) \in\left(\mathbb{R}^{d}\right)^{N}$; we 
will use the notation $\sigma:\left(\mathbb{R}^{d}\right)^{N} \rightarrow\left(\mathbb{R}^{d}\right)^{N}$ to denote a permutation of the coordinates of vectors in $\left(\mathbb{R}^{d}\right)^{N}$ and we set

$$
\mathrm{d}_{N}(\boldsymbol{x}, \boldsymbol{y}):=\min _{\sigma} \frac{1}{N} \sum_{i=1}^{N}\left|x_{i}-\sigma(\boldsymbol{y})_{i}\right|, \quad|\boldsymbol{x}|_{N}:=\mathrm{d}_{N}(\boldsymbol{x}, \boldsymbol{o})=\frac{1}{N} \sum_{i=1}^{N}\left|x_{i}\right| .
$$

To every vector $\boldsymbol{x} \in\left(\mathbb{R}^{d}\right)^{N}$ we can associate the measure $\mu[\boldsymbol{x}]:=\frac{1}{N} \sum_{i=1}^{N} \delta_{x_{i}} \in \mathcal{P}^{N}\left(\mathbb{R}^{d}\right)$ and we notice that by (6.60) [5, Theorem 6.0.1]

$$
\mathrm{d}_{N}(\boldsymbol{x}, \boldsymbol{y})=W_{1}(\mu[\boldsymbol{x}], \mu[\boldsymbol{y}]), \quad|\boldsymbol{x}|_{N}=\int_{\mathbb{R}^{d}}|x| \mathrm{d} \mu[\boldsymbol{x}](x)=W_{1}\left(\mu[\boldsymbol{x}], \delta_{0}\right) .
$$

From now on we say that a map $G^{N}: \mathbb{R}^{d} \times\left(\mathbb{R}^{d}\right)^{N} \rightarrow \mathbb{R}^{k}$ is symmetric if

$$
\boldsymbol{G}^{N}(x, \boldsymbol{y})=\boldsymbol{G}^{N}(x, \sigma(\boldsymbol{y})) \text { for every permutation } \sigma:\left(\mathbb{R}^{d}\right)^{N} \rightarrow\left(\mathbb{R}^{d}\right)^{N} .
$$

Given a symmetric and continuous map $G^{N}$ we can associate a function defined on measures $G^{N}: \mathbb{R}^{d} \times \mathcal{P}^{N}\left(\mathbb{R}^{d}\right) \rightarrow \mathbb{R}^{k}$ by setting

$$
G^{N}(x, \mu[\boldsymbol{y}]):=\boldsymbol{G}^{N}(x, \boldsymbol{y}) .
$$

Throughout the paper we use the following notion of convergence for symmetric maps:

Definition 2.1 We say that a sequence of symmetric maps $G^{N}, N \in \mathbb{N}, \mathcal{P}_{1}$-converges to $G$ : $\mathbb{R}^{d} \times \mathcal{P}_{1}\left(\mathbb{R}^{d}\right) \rightarrow \mathbb{R}^{k}$ uniformly on compact sets as $N \rightarrow+\infty$ if for every sequence of measure $\mu_{k} \in \mathcal{P}^{N_{k}}\left(\mathbb{R}^{d}\right)$ converging to $\mu$ in $\mathcal{P}_{1}\left(\mathbb{R}^{d}\right)$ as $N_{k} \rightarrow \infty$ we have

$$
\lim _{k \rightarrow+\infty} \sup _{x \in C}\left|G^{N_{k}}\left(x, \mu_{k}\right)-G(x, \mu)\right|=0, \quad \text { for every compact } C \subset \mathbb{R}^{d} .
$$

\subsection{Doubling and moderated convex functions}

Definition 2.2 We say that $\phi:[0,+\infty) \rightarrow[0,+\infty)$ is an admissible function if $\phi(0)=0, \phi$ is strictly convex and of class $C^{1}$ with $\phi^{\prime}(0)=0$, superlinear at $+\infty$, and doubling, i.e., there exists $K>0$ such that

$$
\phi(2 r) \leq K(1+\phi(r)) \quad \text { for any } r \in[0,+\infty) .
$$

Let $U$ be a subspace of $\mathbb{R}^{d}$. We say that a convex function $\psi: U \rightarrow[0,+\infty)$ is moderated if there exists an admissible function $\phi:[0,+\infty) \rightarrow[0,+\infty)$ and a constant $C>0$ such that

$$
\phi(|x|)-1 \leq \psi(x) \leq C(1+\phi(|x|)) \quad \text { for every } x \in U .
$$

By convexity, an admissible function $\phi$ satisfies $\phi(r)+\phi^{\prime}(r)(s-r) \leq \phi(s)$ for every $r, s \in[0,+\infty)$; in particular choosing $s=0$ and $s=2 r$ one obtains

$$
0 \leq \phi(r) \leq r \phi^{\prime}(r) \leq(\phi(2 r)-\phi(r)) \leq K(1+\phi(r)) \text { for every } r \in[0,+\infty) .
$$

It is not difficult to see that if a differentiable convex function $\phi$ satisfies

$$
r \phi^{\prime}(r) \leq A(1+\phi(r)) \text { for every } r \geq R,
$$

for some constants $A, R>0$, then $\phi$ satisfies (2.9) with $K=\max \left(\mathrm{e}^{A}, \max _{[0,2 R]} \phi\right)$. In fact, differentiating the function $z \mapsto(\phi(z r)+1)$ for $z \in[1, D]$ and $r \geq R$ we get $\frac{\partial}{\partial \theta}(\phi(\theta r)+1)=$ $r \phi^{\prime}(\theta r) \leq A(1+\phi(\theta r))$ so that

$$
\phi(D r) \leq(\phi(r)+1) \mathrm{e}^{(D-1) A} \quad D>1, r>R .
$$

In particular (2.9) yields

$$
\phi(D r) \leq(\phi(r)+1) \mathrm{e}^{(D-1) K} \quad D>1, r>0 .
$$


We also recall that $\phi^{\prime}$ is monotone, i.e.

$$
\left(\phi^{\prime}(r)-\phi^{\prime}(s)\right)(r-s) \geq 0 \quad \text { for every } s, r \geq 0 .
$$

The next lemma shows that it is always possible to approximate a convex superlinear function by a monotonically increasing sequence of moderated ones.

Lemma 2.3 Let $U$ be a subspace of $\mathbb{R}^{d}$ and $\psi: U \rightarrow[0,+\infty]$ be a superlinear function with $\psi(0)=0$.

1. There exists an admissible function $\theta:[0,+\infty) \rightarrow[0,+\infty)$ such that

$$
\psi(x) \geq \theta(|x|)-\frac{1}{2} \quad \text { for every } x \in U .
$$

2. If $\psi$ is also convex, then there exists a sequence $\psi^{N}: U \rightarrow[0,+\infty), N \in \mathbb{N}$, of moderated convex functions such that

$$
\psi^{N}(x) \leq \psi^{N+1}(x), \quad \psi^{N}(x) \uparrow \psi(x) \quad \text { as } N \rightarrow+\infty \quad \text { for every } x \in U .
$$

Proof. It is not restrictive to assume $U=\mathbb{R}^{d}$.

Claim 1. Let us set $h(r):=\min _{|x| \geq r} \psi(x)$ and $\bar{n}:=\min \left\{n \geq 0: h\left(2^{n}\right) \geq 1\right\}, \bar{r}:=2^{\bar{n}}$. The map $h:[0,+\infty) \rightarrow[0,+\infty]$ is increasing, lower semicontinuous, and satisfies $\lim _{r \rightarrow \infty} h(r) / r=+\infty$. By a standard result of convex analysis (see e.g. [56, Lemma 3.7]) there exists a convex superlinear function $k:[0,+\infty) \rightarrow[0,+\infty)$ such that $h(r) \geq k(r)$ for every $r \in[0,+\infty)$ so that $\psi(x) \geq k(|x|)$ for every $x \in \mathbb{R}^{d}$.

Let us define the sequence $\left(a_{n}\right)_{n \in \mathbb{N}}$ by induction:

$$
\begin{aligned}
a_{n} & :=0 \quad \text { for every } n \in \mathbb{N}, n<\bar{n} ; \quad a_{\bar{n}}:=2^{-\bar{n}}, \\
a_{n+1} & :=\min \left(2 a_{n}, 2^{-(n-1)}\left(k\left(2^{n}\right)-k\left(2^{n-1}\right)\right)\right) \quad \text { for every } n \geq \bar{n} .
\end{aligned}
$$

Since $k$ is convex and increasing, the sequence $n \mapsto a_{n}$ is positive and increasing; since $k$ is superlinear, it is also easy to check that $\lim _{n \rightarrow \infty} a_{n}=+\infty$.

We now consider the piecewise linear continuous function $\theta_{1}:[0,+\infty) \rightarrow[0,+\infty)$ on the dyadic partition $\left\{0,2^{0}, 2^{1}, 2^{2}, \cdots, 2^{n}, \cdots\right\}, n \in \mathbb{N}$, satisfying

$$
\theta_{1}(r) \equiv 0 \quad \text { if } 0 \leq r \leq \bar{r}=2^{\bar{n}}, \quad \theta_{1}^{\prime}(r)=a_{n} \quad \text { if } 2^{n}<r<2^{n+1}, \quad n \in \mathbb{N}, n \geq \bar{n} .
$$

Since $\theta_{1}^{\prime} \leq k^{\prime}$ a.e. in $[0,+\infty)$ we have $\theta_{1} \leq k$. Moreover, by construction, for $0 \leq r \leq 2 \bar{r}$ we have $\theta_{1}(r) \leq \theta_{1}(2 \bar{r})=1$ and $\theta_{1}^{\prime}(2 r) \leq 2 \theta_{1}^{\prime}(r)$ if $r \geq \bar{r}$ so that $\theta_{1}$ is also doubling since

$$
\theta_{1}(2 r)=\theta_{1}(2 \bar{r})+\int_{\bar{r}}^{r} 2 \theta_{1}^{\prime}(2 s) \mathrm{d} s \leq 1+4 \int_{\bar{r}}^{r} \theta_{1}^{\prime}(s) \mathrm{d} s=1+4 \theta_{1}(r) \quad \text { for every } r \geq \bar{r} .
$$

Replacing now $\theta_{1}$ by the convex combination $\theta_{2}(r):=\frac{1}{2} \theta_{1}(r)+\frac{1}{2} r / \bar{r}$ we get a strictly increasing function, still satisfying (2.16).

By possibly replacing $\theta_{2}$ with $\theta_{3}(r):=\int_{r-1}^{r} \theta(s) \mathrm{d} s$ (where we set $\theta_{2}(s) \equiv \theta_{2}(0)=0$ whenever $s<0)$ we obtain a $C^{1}$ function. Strict convexity can be eventually obtained by taking the convex combination $\theta(r):=(1-\varepsilon) \theta_{3}(r)+\varepsilon\left(\sqrt{1+r^{2}}-1\right)$ for a sufficiently small $\varepsilon>0$.

Claim 2. Notice that the function $x \mapsto \theta_{2}(|x|)$ is convex. We can define $\psi^{N}$ by inf-convolution:

$$
\psi^{N}(x):=\inf _{y \in \mathbb{R}^{d}} \psi(y)+N \theta_{2}(|x-y|), \quad x \in \mathbb{R}^{d} .
$$

It is easy to check that the infimum in (2.20) is attained, $\psi^{N}$ is convex (since it is the inf-convolution of two convex functions) and satisfies the obvious bounds

$$
\psi^{N}(x) \leq N \theta_{2}(|x|), \quad \psi^{N}(x) \leq \psi(x), \quad \psi^{N}(x) \leq \psi^{N+1}(x) \quad \text { for every } x \in \mathbb{R}^{d} .
$$


In particular $\psi^{N}$ is continuous; since $x \mapsto \theta_{2}(x)$ is continuous at $x=0$ and $\theta_{2}(|x|) \geq \frac{1}{2 \bar{r}}|x|$ we easily get $\lim _{N \rightarrow \infty} \psi^{N}(x)=\psi(x)$ for every $x \in \mathbb{R}^{d}$.

It remains to show that $\psi^{N}$ is moderated. Since $\psi(x) \geq \theta_{2}(|x|)-1 / 2$ and for every $y \in \mathbb{R}^{d}$ the triangle inequality yields $\min (|x-y|,|y|) \geq|x| / 2$, we get

$$
\psi^{N}(x)+1 / 2 \geq \inf _{y \in \mathbb{R}^{d}} \theta_{2}(|y|)+N \theta_{2}(|x-y|) \geq \theta_{2}(|x| / 2) \geq \frac{1}{4} \theta_{2}(|x|)-\frac{1}{4}
$$

and the bounds

$$
\frac{1}{4} \theta(|x|)-\frac{3}{4} \leq \psi^{N}(x) \leq 4 N \frac{1}{4} \theta_{2}(|x|) .
$$

By possibly replacing $\theta_{2}$ with $\theta$ we conclude.

Let us make explicit two simple applications of the properties of Definition 2.2 .

Remark 2.4 If $\mathcal{K} \subset \mathcal{P}_{1}\left(\mathbb{R}^{d}\right)$ is a relatively compact set and $\psi: U \rightarrow[0,+\infty]$ is a superlinear funcion defined in a subspace $U$ of $\mathbb{R}^{d}$ with $\psi(0)=0$, then there exists an admissible function $\theta:[0,+\infty) \rightarrow[0,+\infty)$ such that

$$
\sup _{\mu \in \mathcal{K}} \int_{\mathbb{R}^{d}} \theta(|x|) \mathrm{d} \mu(x)<\infty, \quad \theta(|x|) \leq 1+\psi(x) \quad \text { for every } x \in U .
$$

In fact, Prokhorov theorem yields the tightness of the set $\tilde{\mathcal{K}}:=\{|x| \mu: \mu \in \mathcal{K}\}$ of finite measures, so that we can find a superlinear function $\alpha: \mathbb{R}^{d} \rightarrow[0, \infty)$ such that

$$
\sup _{\mu \in \mathcal{K}} \int_{\mathbb{R}^{d}} \alpha(x) \mathrm{d} \mu(x)<\infty .
$$

We can then apply the first statement of Lemma 2.3 with superlinear function $\alpha \wedge \psi$.

Lemma 2.5 Let $\zeta: \mathbb{R}^{d} \rightarrow[0,+\infty)$ be a moderated convex function with $\zeta(0)=0$ and let $\mu_{n}^{i} \in$ $\mathcal{P}_{1}\left(\mathbb{R}^{d}\right), i=0,1$, be two sequences converging to $\mu$ in $\mathcal{P}_{1}\left(\mathbb{R}^{d}\right)$ and let $\gamma_{n}$ be the optimal plan attaining the minimum in (2.2) for $W_{1}\left(\mu_{n}^{0}, \mu_{n}^{1}\right)$. If

$$
\limsup _{n \rightarrow \infty} \int \zeta \mathrm{d} \mu_{n}^{i} \leq \int \zeta \mathrm{d} \mu
$$

then

$$
\lim _{n \rightarrow \infty} \int \zeta(y-x) \mathrm{d} \gamma_{n}(x, y)=0, \quad \lim _{n \rightarrow \infty} \mathcal{e}_{\zeta}\left(\mu_{n}^{0}, \mu_{n}^{1}\right)=0 .
$$

Proof. Let $\phi$ be an admissible function satisfying (2.10) for $\psi:=\zeta$. We observe that for every $x, y \in \mathbb{R}^{d}$

$$
\phi(|y-x|) \leq \phi(|x|+|y|) \leq K\left(1+\phi\left(\frac{1}{2}|x|+\frac{1}{2}|y|\right)\right) \leq K(1+\phi(|x|)+\phi(|y|)) .
$$

Inequality (2.26) shows that $\zeta$ is uniformly integrable w.r.t. $\mu_{n}$ (see [5, Lemma 5.1.7]) so that

$$
\lim _{n \rightarrow \infty} \int \phi(|x|) \mathrm{d} \mu_{n}^{i}(x)=\int \phi(|x|) \mathrm{d} \mu(x), \quad i=1,2,
$$

whence

$$
\lim _{n \rightarrow \infty} \int(\phi(|x|)+\phi(|y|)) \mathrm{d} \gamma_{n}(x, y)=2 \int \phi(|x|) \mathrm{d} \mu(x)=\int(\phi(|x|)+\phi(|y|)) \mathrm{d} \gamma(x, y)
$$

where $\gamma:=(x, x)_{\sharp} \mu$ is the weak limit of $\gamma_{n}$. It follows that the function $(x, y) \mapsto \phi(|x|)+\phi(|y|)$ is uniformly integrable with respect to $\gamma_{n}$ so that, by (2.28) and [5, Lemma 5.1.7]

$$
\lim _{n \rightarrow \infty} \int \phi(|y-x|) \mathrm{d} \gamma_{n}(x, y)=\int \phi(|y-x|) \mathrm{d} \gamma(x, y)=0 .
$$

Since $\zeta(y-x) \leq C(1+\phi(|y-x|))$ by (2.10) we get (2.27). 


\subsection{Convex functionals on measures}

We are concerned with the main properties of functionals defined on measures, for a detailed treatment of this subject we refer to [4]. Let $\psi: \mathbb{R}^{h} \rightarrow[0,+\infty]$ be a proper, l.s.c., convex and superlinear function, so that its recession function $\sup _{r>0} \frac{\psi(r x)}{r}=\infty$ for all $x \neq 0$; we will also assume $\psi(0)=0$.

Let now $\Omega$ be an open subset of some Euclidean space $\mu \in \mathcal{M}^{+}(\Omega)$ be a reference measure and $\nu \in \mathcal{M}\left(\Omega ; \mathbb{R}^{h}\right)$ a vector measure; we define the following functional

$$
\Psi(\boldsymbol{\nu} \mid \mu):=\int_{\Omega} \psi(\boldsymbol{v}(x)) \mathrm{d} \mu(x) \quad \text { if } \boldsymbol{\nu}=\boldsymbol{v} \mu \ll \mu, \quad \Psi(\boldsymbol{\nu} \mid \mu):=+\infty \quad \text { if } \boldsymbol{\nu} \nless \mu .
$$

We state the main lower semicontinuity result for the functional $\Psi$.

Theorem 2.6 Suppose that we have two sequences $\mu_{n} \in \mathcal{M}^{+}(\Omega), \nu_{n} \in \mathcal{M}\left(\Omega ; \mathbb{R}^{h}\right)$ weakly converging to $\mu \in \mathcal{M}^{+}(\Omega)$ and $\boldsymbol{\nu} \in \mathcal{M}\left(\Omega, \mathbb{R}^{h}\right)$, respectively. Then

$$
\liminf _{n \rightarrow+\infty} \Psi\left(\boldsymbol{\nu}_{n} \mid \mu_{n}\right) \geq \Psi(\boldsymbol{\nu} \mid \mu)
$$

In particular, if $\liminf _{n \rightarrow+\infty} \Psi\left(\boldsymbol{\nu}_{n} \mid \mu_{n}\right)<+\infty$, we have $\boldsymbol{\nu} \ll \mu$.

The proof can be found in [5], Lemma 9.4.3.

\section{The optimal control problem and main results}

Cost functional. Assume that we are given a sequence of functions $L^{N}: \mathbb{R}^{d} \times\left(\mathbb{R}^{d}\right)^{N} \rightarrow[0,+\infty)$, $N \in \mathbb{N}$, and a function $L: \mathbb{R}^{d} \times \mathcal{P}_{1}\left(\mathbb{R}^{d}\right) \rightarrow[0,+\infty)$ such that $L^{N}$ is continuous and symmetric for every $N \in \mathbb{N}$ and $L$ is continuous. We assume that

$$
L^{N} \mathcal{P}_{1} \text {-converges to } L \text { uniformly on compact sets, as } N \rightarrow \infty \text {, }
$$

in the sense of Definition 2.1.

Assume that we are given

a subspace $U \subset \mathbb{R}^{d}$ and a moderated convex function $\psi: U \rightarrow[0,+\infty)$ with $\psi(0)=0$.

We will also fix an auxiliary function $\phi$ satisfying (2.10).

Typical examples we consider for $\psi$ include

- $\psi(x)=\frac{1}{p}|x|^{p}, \quad p>1$;

- $\psi(x)=\frac{1}{p}|x|$ for $|x| \leq 1$ and $\psi(x)=\frac{1}{p}|x|^{p}$ for $|x|>1, p>1$.

Denoting by $U^{N}$ the Cartesian product, we define a cost functional $\mathcal{E}^{N}: A C\left([0, T] ;\left(\mathbb{R}^{d}\right)^{N}\right) \times$ $L^{1}\left([0, T] ; U^{N}\right) \rightarrow[0,+\infty)$ by

$$
\mathcal{E}^{N}(\boldsymbol{x}, \boldsymbol{u}):=f_{0}^{T} \frac{1}{N} \sum_{i=1}^{N} L^{N}\left(x_{i}(t), \boldsymbol{x}(t)\right) \mathrm{d} t+f_{0}^{T} \frac{1}{N} \sum_{i=1}^{N} \psi\left(u_{i}(t)\right) \mathrm{d} t .
$$

We consider also another cost functional $\mathcal{E}: A C\left([0, T] ; \mathcal{P}_{1}\left(\mathbb{R}^{d}\right)\right) \times \mathcal{M}\left([0, T] \times \mathbb{R}^{d} ; U\right) \rightarrow[0,+\infty)$ defined by (recall (2.4))

$$
\mathcal{E}(\mu, \boldsymbol{\nu}):=f_{0}^{T} \int_{\mathbb{R}^{d}} L\left(x, \mu_{t}\right) \mathrm{d} \mu_{t}(x) \mathrm{d} t+\Psi(\boldsymbol{\nu} \mid \tilde{\mu}),
$$

where $\Psi$ is defined as in (2.32). Notice that if $\Psi(\boldsymbol{\nu} \mid \tilde{\mu})<\infty$ then $\boldsymbol{\nu}=\boldsymbol{v} \mu$ for a Borel vector field $\boldsymbol{v} \in L_{\tilde{\mu}}^{1}\left([0, T] \times \mathbb{R}^{d} ; U\right)$ so that for $\lambda$-a.e. $t \in[0, T]$ the measure $\boldsymbol{\nu}_{t}:=\boldsymbol{v}(t, \cdot) \mu_{t}$ belongs to $\mathcal{M}\left(\mathbb{R}^{d} ; U\right)$ and we can write

$$
\Psi(\boldsymbol{\nu} \mid \tilde{\mu})=\int_{[0, T] \times \mathbb{R}^{d}} \psi(\boldsymbol{v}(t, x)) \mathrm{d} \tilde{\mu}(t, x)=f_{0}^{T} \int_{\mathbb{R}^{d}} \psi(\boldsymbol{v}(t, x)) \mathrm{d} \mu_{t} \mathrm{~d} t=f_{0}^{T} \Psi\left(\boldsymbol{\nu}_{t} \mid \mu_{t}\right) \mathrm{d} t .
$$

We shall prove below that the functional $\mathcal{E}$ is the $\Gamma$-limit of $\mathcal{E}^{N}$ in suitable sense [34]. 
The constraints (State equations). Assume that we are given a sequence of functions $\boldsymbol{F}^{N}$ : $\mathbb{R}^{d} \times\left(\mathbb{R}^{d}\right)^{N} \rightarrow \mathbb{R}^{d}, N \in \mathbb{N}$, symmetric and continuous and a continuous function $\boldsymbol{F}: \mathbb{R}^{d} \times \mathcal{P}_{1}\left(\mathbb{R}^{d}\right) \rightarrow$ $\mathbb{R}^{d}$. We assume that there exist constants $A, B \geq 0$ such that

$$
\left|\boldsymbol{F}^{N}(x, \boldsymbol{y})\right| \leq A+B\left(|x|+|\boldsymbol{y}|_{N}\right), \quad|\boldsymbol{F}(x, \mu)| \leq A+B\left(|x|+\int_{\mathbb{R}^{d}}|y| \mathrm{d} \mu(y)\right),
$$

and $\boldsymbol{F}^{N}, \boldsymbol{F}$ and $U$ satisfy the compatibility condition

$$
\boldsymbol{F}^{N}(x, \boldsymbol{y})-\boldsymbol{F}(x, \mu) \in U \quad \text { for every } x \in \mathbb{R}^{d}, \boldsymbol{y} \in\left(\mathbb{R}^{d}\right)^{N}, \mu \in \mathcal{P}_{1}\left(\mathbb{R}^{d}\right) .
$$

Moreover, we assume that

$$
\boldsymbol{F}^{N} \mathcal{P}_{1} \text {-converges to } \boldsymbol{F} \text { uniformly on compact sets, as } N \rightarrow \infty,
$$

in the sense of Definition 2.1.

Given $\boldsymbol{u}=\left(u_{1}, \ldots, u_{N}\right) \in L^{1}\left([0, T] ; U^{N}\right)$, a control map, we consider the system of differential equations

$$
\dot{x}_{i}(t)=\boldsymbol{F}^{N}\left(x_{i}(t), \boldsymbol{x}(t)\right)+u_{i}(t), \quad i=1, \cdots, N .
$$

The map $\boldsymbol{F}^{N}: \mathbb{R}^{d} \times\left(\mathbb{R}^{d}\right)^{N} \rightarrow \mathbb{R}^{d}$ models the interaction between the agents and $\boldsymbol{u}$ represents the action of an external controller on the system. For every $\boldsymbol{u} \in L^{1}\left([0, T] ; U^{N}\right)$ and $\boldsymbol{x}_{0} \in\left(\mathbb{R}^{d}\right)^{N}$, thanks to (3.6) and the continuity of $\boldsymbol{F}^{N}$, there exists a global solution, in the Carathéodory sense, $\boldsymbol{x} \in A C\left([0, T] ;\left(\mathbb{R}^{d}\right)^{N}\right)$ of (3.9) such that $\boldsymbol{x}(0)=\boldsymbol{x}_{0}$. Since we have assumed only the continuity of the velocity field $\boldsymbol{F}^{N}$, uniqueness of solutions is not guaranteed in general. We then define the non empty set

$$
\mathscr{A}^{N}:=\left\{(\boldsymbol{x}, \boldsymbol{u}) \in A C\left([0, T] ;\left(\mathbb{R}^{d}\right)^{N} \times L^{1}\left([0, T] ; U^{N}\right): \boldsymbol{x} \text { and } \boldsymbol{u} \text { satisfy (3.9)}\right), \mathcal{E}^{N}(\boldsymbol{x}, \boldsymbol{u})<\infty\right\} .
$$

Moreover we also define for every $\boldsymbol{x}_{0} \in\left(\mathbb{R}^{d}\right)^{N}$ the non empty set

$$
\mathscr{A}^{N}\left(\boldsymbol{x}_{0}\right):=\left\{(\boldsymbol{x}, \boldsymbol{u}) \in \mathscr{A}^{N}: \boldsymbol{x}(0)=\boldsymbol{x}_{0}\right\} .
$$

Every initial vector $\boldsymbol{x}_{0}=\left(x_{0,1}, \cdots, x_{0, N}\right) \in\left(\mathbb{R}^{d}\right)^{N}$ gives raise to the empirical distribution

$$
\mu_{0}=\mu\left[\boldsymbol{x}_{0}\right]:=\frac{1}{N} \sum_{i=1}^{N} \delta_{x_{0, i}} .
$$

Similarly, every curve $\boldsymbol{x} \in A C\left([0, T] ;\left(\mathbb{R}^{d}\right)^{N}\right)$ is associated to the curve of probability measures

$$
\mu=\mu[\boldsymbol{x}] \in A C\left([0, T] ; \mathcal{P}_{1}\left(\mathbb{R}^{d}\right)\right): \quad \mu_{t}:=\frac{1}{N} \sum_{i=1}^{N} \delta_{x_{i}(t)}, \quad t \in[0, T],
$$

and every pair $(\boldsymbol{x}, \boldsymbol{u}) \in A C\left([0, T] ;\left(\mathbb{R}^{d}\right)^{N}\right) \times L^{1}\left([0, T] ; U^{N}\right)$ is linked to the control vector measure

$$
\boldsymbol{\nu}=\boldsymbol{\nu}[\boldsymbol{x}, \boldsymbol{u}] \in \mathcal{M}\left([0, T] \times \mathbb{R}^{d} ; U\right): \quad \boldsymbol{\nu}:=\int_{0}^{T} \delta_{t} \otimes \boldsymbol{\nu}_{t} \mathrm{~d} \lambda, \quad \boldsymbol{\nu}_{t}:=\frac{1}{N} \sum_{i=1}^{N} u_{i}(t) \delta_{x_{i}(t)} .
$$

We will show that for every choice of solutions and controls $\left(\boldsymbol{x}^{N}, \boldsymbol{u}^{N}\right) \in \mathscr{A}^{N}\left(\boldsymbol{x}_{0}^{N}\right)$ such that the cost functional $\mathcal{E}^{N}\left(\boldsymbol{x}^{N}, \boldsymbol{u}^{N}\right)$ remains uniformly bounded and the initial empirical distributions $\mu_{0}^{N}=\mu\left[\boldsymbol{x}_{0}^{N}\right]$ is converging to a limit measure $\mu_{0}$ in $\mathcal{P}_{1}\left(\mathbb{R}^{d}\right)$ a mean-field approximation holds:

Theorem 3.1 (Compactness) Let $\left(\boldsymbol{x}_{0}^{N}\right)_{N \in \mathbb{N}}$ be a sequence of initial data in $\left(\mathbb{R}^{d}\right)^{N}$ such that the empirical measure $\mu_{0}^{N}=\mu\left[\boldsymbol{x}_{0}^{N}\right]$ converges to a probability measure $\mu_{0}$ in $\mathcal{P}_{1}\left(\mathbb{R}^{d}\right)$ as $N \rightarrow \infty$, and let $\left(\boldsymbol{x}^{N}, \boldsymbol{u}^{N}\right) \in \mathscr{A}^{N}\left(\boldsymbol{x}_{0}^{N}\right)$ such that the cost functional $\mathcal{E}^{N}\left(\boldsymbol{x}^{N}, \boldsymbol{u}^{N}\right)$ remains uniformly bounded. Up to extraction of a suitable subsequence, the empirical measures $\mu^{N}=\mu\left[\boldsymbol{x}^{N}\right]$ converge uniformly 
in $\mathcal{P}_{1}\left(\mathbb{R}^{d}\right)$ to a curve of probability measures $\mu \in A C\left([0, T] ; \mathcal{P}_{1}\left(\mathbb{R}^{d}\right)\right)$, the control measures $\boldsymbol{\nu}^{N}=$ $\boldsymbol{\nu}\left[\boldsymbol{x}^{N}, \boldsymbol{u}^{N}\right]$ converge to a limit control measure $\boldsymbol{\nu}$ weakly ${ }^{*}$ in $\mathcal{M}\left([0, T] \times \mathbb{R}^{d} ; U\right)$, and $(\mu, \boldsymbol{\nu})$ fulfills the continuity equation

$$
\partial_{t} \mu_{t}+\nabla \cdot\left(\boldsymbol{F}\left(x, \mu_{t}\right) \mu_{t}+\boldsymbol{\nu}_{t}\right)=0 \quad \text { in }(0, T) \times \mathbb{R}^{d}
$$

in the sense of distributions.

Motivated by the above result, we define the non empty set

$$
\begin{aligned}
& \mathscr{A}:=\left\{(\mu, \boldsymbol{\nu}) \in A C\left([0, T] ; \mathcal{P}_{1}\left(\mathbb{R}^{d}\right)\right) \times \mathcal{M}\left([0, T] \times \mathbb{R}^{d} ; U\right):\right. \\
&\mu \text { and } \boldsymbol{\nu} \text { satisfy (13.13) } \text { in the sense of distributions, } \mathcal{E}(\mu, \boldsymbol{\nu})<\infty\},
\end{aligned}
$$

and its corresponding subset associated to a given initial measure $\mu_{0} \in \mathcal{P}_{1}\left(\mathbb{R}^{d}\right)$ :

$$
\mathscr{A}\left(\mu_{0}\right):=\left\{(\mu, \nu) \in \mathscr{A}: \mu(0)=\mu_{0}\right\} .
$$

The elements of $\mathscr{A}^{N}$ can be interpreted as the trajectories $\left(x_{1}, \ldots, x_{N}\right)$ of $N$ agents along with their strategies $\left(u_{1}, \ldots, u_{N}\right)$, whose dynamics is described by the system of ODEs (3.9). Analogously, the elements of $\mathscr{A}$ can be interpreted as the trajectories of a continuous or discrete distribution of agents whose dynamics is described by the PDE (3.13) under the action of an external controller described by the measure $\boldsymbol{\nu}$.

The minimum problems. The objective of the controller is to minimize the cost functional $\mathcal{E}^{N}$ (resp. $\mathcal{E}$ ). We consider the following optimum sets, defined by corresponding optimal control problems:

$$
\begin{aligned}
E^{N}\left(\boldsymbol{x}_{0}\right) & :=\min _{(\boldsymbol{x}, \boldsymbol{u}) \in \mathscr{A}^{N}\left(\boldsymbol{x}_{0}\right)} \mathcal{E}^{N}(\boldsymbol{x}, \boldsymbol{u}), & P^{N}\left(\boldsymbol{x}_{0}\right):=\operatorname{argmin}\left\{\mathcal{E}^{N}(\boldsymbol{x}, \boldsymbol{u}):(\boldsymbol{x}, \boldsymbol{u}) \in \mathscr{A}^{N}\left(\boldsymbol{x}_{0}\right)\right\}, \\
E\left(\mu_{0}\right) & :=\min _{(\mu, \boldsymbol{\nu}) \in \mathscr{A}\left(\mu_{0}\right)} \mathcal{E}(\mu, \boldsymbol{\nu}), & P\left(\mu_{0}\right):=\operatorname{argmin}\left\{\mathcal{E}(\mu, \boldsymbol{\nu}):(\mu, \boldsymbol{\nu}) \in \mathscr{A}\left(\mu_{0}\right)\right\},
\end{aligned}
$$

where we suppose that $\mu_{0} \in D(E):=\left\{\mu \in \mathcal{P}_{1}\left(\mathbb{R}^{d}\right): \mathscr{A}(\mu)\right.$ is not empty $\}$.

We are interested in the rigorous justification of the convergence of the control problem (3.14) towards the corresponding infinite dimensional one (3.15).

Main results. We state now more formally our main result concerning the sequence of functionals $\mathcal{E}^{N}$ to $\mathcal{E}$, inspired to $\Gamma$-convergence.

Theorem 3.2 ( $\Gamma$-convergence) The following properties hold:

- $\Gamma$ - liminf inequality: for every $(\mu, \boldsymbol{\nu}) \in A C\left([0, T] ; \mathcal{P}_{1}\left(\mathbb{R}^{d}\right)\right) \times \mathcal{M}\left([0, T] \times \mathbb{R}^{d} ; U\right)$ and every sequence $\left(\boldsymbol{x}^{N}, \boldsymbol{u}^{N}\right) \in A C\left([0, T] ;\left(\mathbb{R}^{d}\right)^{N}\right) \times L^{1}\left([0, T] ; U^{N}\right)$ such that $\mu\left[\boldsymbol{x}^{N}\right] \rightarrow \mu$ in $C\left([0, T] ; \mathcal{P}_{1}\left(\mathbb{R}^{d}\right)\right)$, $\boldsymbol{\nu}\left[\boldsymbol{x}^{N}, \boldsymbol{u}^{N}\right] \boldsymbol{\rightarrow}^{*} \boldsymbol{\nu}$ in $\mathcal{M}\left([0, T] \times \mathbb{R}^{d} ; U\right)$, we have

$$
\liminf _{N \rightarrow \infty} \mathcal{E}^{N}\left(\boldsymbol{x}^{N}, \boldsymbol{u}^{N}\right) \geq \mathcal{E}(\mu, \boldsymbol{\nu}) .
$$

- $\Gamma$ - limsup inequality: for every $(\mu, \boldsymbol{\nu}) \in \mathscr{A}$ such that

$$
\int_{\mathbb{R}^{d}} \phi(|x|) \mathrm{d} \mu_{0}(x)<\infty
$$

there exists a sequence $\left(\boldsymbol{x}^{N}, \boldsymbol{u}^{N}\right) \in \mathscr{A}^{N}$ with $x_{0, i}^{N} \in \operatorname{supp}\left(\mu_{0}\right)$ for every $i=1, \cdots, N$, such that

$$
\begin{gathered}
\mu\left[\boldsymbol{x}^{N}\right] \rightarrow \mu \text { in } C\left([0, T] ; \mathcal{P}_{1}\left(\mathbb{R}^{d}\right)\right), \quad \boldsymbol{\nu}\left[\boldsymbol{x}^{N}, \boldsymbol{u}^{N}\right]-^{*} \boldsymbol{\nu} \text { in } \mathcal{M}\left([0, T] \times \mathbb{R}^{d} ; U\right), \\
\lim _{N \rightarrow \infty} \frac{1}{N} \sum_{i=1}^{N} \phi\left(\left|x_{0, i}^{N}\right|\right)=\int_{\mathbb{R}^{d}} \phi(|x|) \mathrm{d} \mu_{0}(x),
\end{gathered}
$$


and

$$
\limsup _{N \rightarrow \infty} \mathcal{E}^{N}\left(\boldsymbol{x}^{N}, \boldsymbol{u}^{N}\right) \leq \mathcal{E}(\mu, \boldsymbol{\nu})
$$

As a combination of Theorem 3.1 and Theorem 3.2 we obtain the convergence of minima.

Theorem 3.3 Let $\mu_{0} \in \mathcal{P}_{1}\left(\mathbb{R}^{d}\right)$ be satisfying (3.17).

1. There exists a sequence $\boldsymbol{x}_{0}^{N} \in\left(\mathbb{R}^{d}\right)^{N}, N \in \mathbb{N}$, satisfying

$$
\begin{gathered}
\lim _{N \rightarrow \infty} W_{1}\left(\mu\left[\boldsymbol{x}_{0}^{N}\right], \mu_{0}\right)=0, \\
\limsup _{N \rightarrow \infty} \frac{1}{N} \sum_{i=1}^{N} \phi\left(\left|x_{0, i}^{N}\right|\right)=\int \phi(|x|) \mathrm{d} \mu_{0}(x), \\
\lim _{N \rightarrow \infty} E^{N}\left(\boldsymbol{x}_{0}^{N}\right)=E\left(\mu_{0}\right) .
\end{gathered}
$$

2. If a sequence $\boldsymbol{x}_{0}^{N}$ satisfies (3.21) then for every choice of $\left(\boldsymbol{x}^{N}, \boldsymbol{u}^{N}\right) \in P\left(\boldsymbol{x}_{0}^{N}\right)$ with $\mu^{N}:=$ $\mu\left[\boldsymbol{x}^{N}\right]$ and $\boldsymbol{\nu}^{N}:=\boldsymbol{\nu}\left[\boldsymbol{x}^{N}, \boldsymbol{u}^{N}\right]$, the collection of limit points $(\mu, \boldsymbol{\nu})$ of $\left(\mu^{N}, \boldsymbol{\nu}^{N}\right)$ in $C\left([0, T] ; \mathcal{P}_{1}\left(\mathbb{R}^{d}\right)\right) \times$ $\mathcal{M}\left([0, T] \times \mathbb{R}^{d} ; U\right)$ is non empty and contained in $P\left(\mu_{0}\right)$.

3. If moreover $U=\mathbb{R}^{d}$ and $\mu_{0}$ has compact support, then every sequence $\left(\boldsymbol{x}_{0}^{N}\right)_{N \in \mathbb{N}}$ satisfying (3.21) and uniformly supported in a compact set also satisfies (3.22) and (3.23).

\subsection{Examples}

First order examples. Take a continuous function $H: \mathbb{R}^{d} \rightarrow \mathbb{R}^{d}$ satysfying

$$
|H(x)| \leq A+B|x| \quad \forall x \in \mathbb{R}^{d}
$$

and set

$$
\boldsymbol{F}^{N}(x, \boldsymbol{y}):=\frac{1}{N} \sum_{j=1}^{N} H\left(x-y_{j}\right)=\int_{\mathbb{R}^{d}} H(x-y) \mathrm{d} \mu[\boldsymbol{y}](y)
$$

and

$$
\boldsymbol{F}(x, \mu):=\int_{\mathbb{R}^{d}} H(x-y) \mathrm{d} \mu(y) .
$$

When $H=-\nabla W$ for an even function $W \in C^{1}\left(\mathbb{R}^{d}\right)$ the system (3.9) is associated to the gradient flow of the interaction energy $\mathcal{W}:\left(\mathbb{R}^{d}\right)^{N} \rightarrow \mathbb{R}$ defined by

$$
\mathcal{W}(\boldsymbol{x}):=\frac{1}{2 N^{2}} \sum_{i, j=1}^{N} W\left(x_{i}-x_{j}\right)
$$

with respect to the weighted norm $\|\boldsymbol{x}\|^{2}=\frac{1}{N} \sum_{i=1}^{N}\left|x_{i}\right|^{2}$.

More generally, we can consider a continuous kernel $K(x, y): \mathbb{R}^{d} \times \mathbb{R}^{d} \rightarrow \mathbb{R}^{d}$ satisfying

$$
|K(x, y)| \leq A+B(|x|+|y|) \quad \forall x, y \in \mathbb{R}^{d}
$$

obtaining

$$
\boldsymbol{F}^{N}(x, \boldsymbol{y}):=\frac{1}{N} \sum_{j=1}^{N} K\left(x, y_{j}\right)=\int_{\mathbb{R}^{d}} K(x, y) \mathrm{d} \mu[\boldsymbol{y}](y)
$$

and

$$
\boldsymbol{F}(x, \mu):=\int_{\mathbb{R}^{d}} K(x, y) \mathrm{d} \mu(y)
$$


An example for $L^{N}$ and $L$ is the variance:

$$
L^{N}(x, \boldsymbol{x}):=\left|x-\frac{1}{N} \sum_{j=1}^{N} x_{j}\right|^{2}
$$

and

$$
L(x, \mu):=\left|x-\int_{\mathbb{R}^{d}} y \mathrm{~d} \mu(y)\right|^{2} .
$$

A second order example. Second order systems can be easily reduced to first order models, if we admit controls on positions and velocities. Let us see an example where controls act only on the velocities. Assume $d=2 m$ and write the vector $x=(q, p)$, where $q \in \mathbb{R}^{m}$ denotes the position and $p \in \mathbb{R}^{m}$ the velocity.

We consider the vector field $\boldsymbol{F}^{N}(x, \boldsymbol{x})=\left(\boldsymbol{F}_{1}^{N}(x), \boldsymbol{F}_{2}^{N}(x, \boldsymbol{x})\right)$ defined by

$$
\boldsymbol{F}_{1}^{N}((q, p))=p, \quad \boldsymbol{F}_{2}^{N}((q, p),(\boldsymbol{q}, \boldsymbol{p}))=-\frac{1}{N} \sum_{j=1}^{N} \nabla W\left(p-p_{j}\right),
$$

where the first component $\boldsymbol{F}_{1}^{N}$ is local and it is not influenced by the interaction with the other particles.

We are interested to the system

$$
\left\{\begin{array}{l}
\dot{q}_{i}=p_{i} \\
\dot{p}_{i}=-\frac{1}{N} \sum_{j=1}^{N} \nabla W\left(p_{i}-p_{j}\right)+u_{i}
\end{array}\right.
$$

which corresponds to (3.9) where the vector $\boldsymbol{u}$ has the particular form $\boldsymbol{u}=\left(\left(0, u_{1}\right), \cdots,\left(0, u_{N}\right)\right)$, so that it is constrained to the subspace $U^{N}$ where $U=\left\{(0, u): u \in \mathbb{R}^{m}\right\} \subset \mathbb{R}^{2 m}$. The limit vector field $\boldsymbol{F}(x, \mu)=\left(\boldsymbol{F}_{1}(x), \boldsymbol{F}_{2}(x, \mu)\right)$ is defined by

$$
\boldsymbol{F}_{1}((q, p))=p, \quad \boldsymbol{F}_{2}((q, p), \mu)=-\nabla_{p} W * \mu,
$$

and the continuity equation

$$
\partial_{t} \mu_{t}+\nabla \cdot\left(\boldsymbol{F}\left(x, \mu_{t}\right) \mu_{t}+\boldsymbol{\nu}_{t}\right)=0
$$

becomes a Vlasov-like equation

$$
\partial_{t} \mu_{t}+p \cdot \nabla_{q} \mu_{t}+\nabla_{p} \cdot\left(\boldsymbol{F}_{2}\left(x, \mu_{t}\right) \mu_{t}+\boldsymbol{\nu}_{t}\right)=0 .
$$

It is easy to check that this structure fits in our abstract setting, since $\boldsymbol{F}^{N}, \boldsymbol{F}$ satisfy the compatibility condition (3.7): for every $x \in \mathbb{R}^{d}, \boldsymbol{y} \in\left(\mathbb{R}^{d}\right)^{N}$ and $\mu \in \mathcal{P}_{1}\left(\mathbb{R}^{d}\right)$ we have $\boldsymbol{F}(x, \mu)-\boldsymbol{F}^{N}(x, \boldsymbol{y})=$ $\left(0, \boldsymbol{F}_{2}(x, \mu)-\boldsymbol{F}_{2}^{N}(x, \boldsymbol{y})\right) \in U^{N}$.

By choosing in (3.29)

$$
\boldsymbol{F}_{2}^{N}((q, p),(\boldsymbol{q}, \boldsymbol{p}))=-\alpha p-\frac{1}{N} \sum_{j=1}^{N} \nabla W\left(p-p_{j}\right)
$$

for some $\alpha>0$ we obtain a model with friction in the velocity part. By choosing in (3.29)

$$
\boldsymbol{F}_{2}^{N}((q, p),(\boldsymbol{q}, \boldsymbol{p}))=-\frac{1}{N} \sum_{j=1}^{N} a\left(\left|q-q_{j}\right|\right)\left(p-p_{j}\right)
$$


where $a:[0,+\infty) \rightarrow \mathbb{R}_{+}$is a continuous and nonincreasing (thus bounded) function, we obtain a model of alignment. A particular and interesting example for $a$ is given by the following decreasing function $a(|q|)=1 /\left(1+|q|^{2}\right)^{\gamma}$ for some $\gamma \geq 0$, which yields the Cucker-Smale flocking model [3132].

An example for $L^{N}$ and $L$ in the second order model is the variance of the velocities:

$$
L^{N}((q, p),(\boldsymbol{q}, \boldsymbol{p})):=\left|p-\frac{1}{N} \sum_{j=1}^{N} p_{j}\right|^{2},
$$

and

$$
L((q, p), \mu):=\left|p-\int_{\mathbb{R}^{d}} r_{2} \mathrm{~d} \mu\left(r_{1}, r_{2}\right)\right|^{2}
$$

\section{The finite dimensional problem}

Here we discuss the well-posedness of the finite dimensional control problem (3.14).

A first estimate on the solution is presented in the following Lemma, where we use the notation $|\boldsymbol{y}|_{N}=\frac{1}{N} \sum_{i=1}^{N}\left|y_{i}\right|$, with $\boldsymbol{y}=\left(y_{1}, \ldots, y_{N}\right) \in\left(\mathbb{R}^{d}\right)^{N}$.

Lemma 4.1 Let $(\boldsymbol{x}, \boldsymbol{u}) \in \mathscr{A}^{N}$. Then

$$
\sup _{t \in[0, T]}|\boldsymbol{x}(t)|_{N} \leq\left(|\boldsymbol{x}(0)|_{N}+A T+\int_{0}^{T}|\boldsymbol{u}(s)|_{N} \mathrm{~d} s\right) e^{2 B T},
$$

where $A$ and $B$ are the constants of the assumption (3.6).

Proof. From the integral formulation of equation (3.9) we get

$$
\begin{aligned}
\left|x_{i}(t)\right| & \leq\left|x_{i}(0)\right|+\int_{0}^{t}\left|\boldsymbol{F}^{N}\left(x_{i}(s), \boldsymbol{x}(s)\right)\right| \mathrm{d} s+\int_{0}^{t}\left|u_{i}(s)\right| \mathrm{d} s \\
& \leq\left|x_{i}(0)\right|+\int_{0}^{t}\left(A+B\left(\left|x_{i}(s)\right|+|\boldsymbol{x}(s)|_{N}\right)\right) \mathrm{d} s+\int_{0}^{t}\left|u_{i}(s)\right| \mathrm{d} s .
\end{aligned}
$$

Averaging with respect to $N$ we obtain

$$
|\boldsymbol{x}(t)|_{N} \leq|\boldsymbol{x}(0)|_{N}+A T+\int_{0}^{T}|\boldsymbol{u}(s)|_{N} \mathrm{~d} s+2 B \int_{0}^{t}|\boldsymbol{x}(s)|_{N} \mathrm{~d} s
$$

and we conclude by Gronwall lemma.

Proposition 4.2 For every $N \in \mathbb{N}$ and $\boldsymbol{x}_{0} \in\left(\mathbb{R}^{d}\right)^{N}$ the minimum problem (3.14) admits a solution, i.e., the set $P^{N}\left(\boldsymbol{x}_{0}\right)$ is not empty.

Proof. We fix $N \in \mathbb{N}$ and $\boldsymbol{x}_{0} \in\left(\mathbb{R}^{d}\right)^{N}$. Let $\lambda:=\inf \left\{\mathcal{E}^{N}(\boldsymbol{x}, \boldsymbol{u}):(\boldsymbol{x}, \boldsymbol{u}) \in \mathscr{A}^{N}\left(\boldsymbol{x}_{0}\right)\right\}$. Since $\mathscr{A}^{N}\left(\boldsymbol{x}_{0}\right)$ is not empty, $\lambda<+\infty$. Let $\left(\boldsymbol{x}^{k}, \boldsymbol{u}^{k}\right) \in \mathscr{A}^{N}\left(\boldsymbol{x}_{0}\right)$ be a minimizing sequence and $C:=$ $\sup _{k} \mathcal{E}^{N}\left(\boldsymbol{x}^{k}, \boldsymbol{u}^{k}\right)<+\infty$.

Since

$$
\sup _{k} f_{0}^{T} \psi\left(u_{i}^{k}(t)\right) \mathrm{d} t \leq C, \quad \forall i=1, \ldots, N,
$$

and the function $\psi$ is superlinear, then the sequence $\boldsymbol{u}^{k}$ is equi-integrable and hence weakly relatively compact in $L^{1}\left([0, T] ; U^{N}\right)$. Hence there exists $\boldsymbol{u} \in L^{1}\left([0, T], U^{N}\right)$ and a subsequence, again denoted by $\boldsymbol{u}^{k}$, weakly convergent to $\boldsymbol{u}$ in $L^{1}\left([0, T], U^{N}\right)$. 
Thanks to Lemma 4.1 the associated trajectories $\boldsymbol{x}^{k}$ are equi-bounded. Let us now show the equi-continuity of $x_{i}^{k}(t)$. For $s \leq t$, by the equation (3.9) we have

$$
x_{i}^{k}(t)-x_{i}^{k}(s)=\int_{s}^{t} \boldsymbol{F}^{N}\left(x_{i}^{k}(r), \boldsymbol{x}^{k}(r)\right) \mathrm{d} r+\int_{s}^{t} u_{i}^{k}(r) \mathrm{d} r .
$$

Using the growth condition (3.6) and (4.1) we get

$$
\begin{aligned}
\left|\boldsymbol{x}^{k}(t)-\boldsymbol{x}^{k}(s)\right|_{N} & \leq \frac{1}{N} \sum_{i=1}^{N} \int_{s}^{t}\left|\boldsymbol{F}^{N}\left(x_{i}^{k}(r), \boldsymbol{x}^{k}(r)\right)\right| \mathrm{d} r+\int_{s}^{t}\left|\boldsymbol{u}^{k}(r)\right|_{N} \mathrm{~d} r \\
& \leq A(t-s)+2 B \int_{s}^{t}\left|\boldsymbol{x}^{k}(r)\right|_{N} \mathrm{~d} r+\int_{s}^{t}\left|\boldsymbol{u}^{k}(r)\right|_{N} \mathrm{~d} r \\
& \leq A(t-s)+2 B\left(\left|\boldsymbol{x}_{0}\right|_{N}+A T+\int_{0}^{T}\left|\boldsymbol{u}^{k}(r)\right|_{N} \mathrm{~d} r\right) e^{2 B T}(t-s)+\int_{s}^{t}\left|\boldsymbol{u}^{k}(r)\right|_{N} \mathrm{~d} r .
\end{aligned}
$$

Since $\int_{0}^{T}\left|\boldsymbol{u}^{k}(r)\right|_{N} \mathrm{~d} r$ is bounded, we have

$$
\sup _{k}\left|\boldsymbol{x}^{k}(t)-\boldsymbol{x}^{k}(s)\right|_{N} \leq \tilde{C}|t-s|+\left.\sup _{k}\left|\int_{s}^{t}\right| \boldsymbol{u}^{k}(r)\right|_{N} \mathrm{~d} r \mid, \quad \forall s, t \in[0, T],
$$

where $\tilde{C}:=A+2 B\left(\left|\boldsymbol{x}_{0}\right|_{N}+A T+\sup _{k} \int_{0}^{T}\left|\boldsymbol{u}^{k}(r)\right|_{N} \mathrm{~d} r\right) e^{2 B T}$. By the equi-integrability of $\boldsymbol{u}^{k}$, the inequality (4.6) shows the equi-continuity of $x^{k}$. By Ascoli-Arzelà theorem there exists a continuous curve $\boldsymbol{x}$ and a subsequence, again denoted by $\boldsymbol{x}^{k}$ such that $\boldsymbol{x}^{k} \rightarrow \boldsymbol{x}$ in $C\left([0, T] ;\left(\mathbb{R}^{d}\right)^{N}\right)$. Passing to the limit in (4.5) we obtain

$$
x_{i}(t)-x_{i}(s)=\int_{s}^{t} \boldsymbol{F}^{N}\left(x_{i}(r), \boldsymbol{x}(r)\right) \mathrm{d} r+\int_{s}^{t} u_{i}(r) \mathrm{d} r, \quad i=1, \ldots, N,
$$

from which we deduce that $\boldsymbol{x}$ is absolutely continuous and solves the equation (3.9). Hence $(\boldsymbol{x}, \boldsymbol{u}) \in \mathscr{A}^{N}\left(\boldsymbol{x}_{0}\right)$.

Finally, by the convexity of $\psi$ and the continuity of $L^{N}$ we obtain the lower semicontinuity property

$$
\begin{aligned}
\liminf _{k} \mathcal{E}^{N}\left(\boldsymbol{x}^{k}, \boldsymbol{u}^{k}\right) & =\liminf _{k}\left[f_{0}^{T} \frac{1}{N} \sum_{i=1}^{N} L^{N}\left(x_{i}^{k}(t), \boldsymbol{x}^{k}(t)\right) \mathrm{d} t+\frac{1}{N} \sum_{i=1}^{N} f_{0}^{T} \psi\left(u_{i}^{k}(t)\right) \mathrm{d} t\right] \\
& \geq f_{0}^{T} \frac{1}{N} \sum_{i=1}^{N} L^{N}\left(x_{i}(t), \boldsymbol{x}(t)\right) \mathrm{d} t+\frac{1}{N} \sum_{i=1}^{N} f_{0}^{T} \psi\left(u_{i}(t)\right) \mathrm{d} t
\end{aligned}
$$

whence the minimality of $(\boldsymbol{x}, \boldsymbol{u}) \in \mathscr{A}^{N}\left(\boldsymbol{x}_{0}\right)$.

\section{$5 \quad$ Momentum estimates}

In this section we study the set $\mathscr{A}$. We observe that if $(\mu, \boldsymbol{\nu}) \in \mathscr{A}$, then for any $\zeta \in C_{c}^{1}\left(\mathbb{R}^{d}\right)$ we have that the map $t \mapsto \int_{\mathbb{R}^{d}} \zeta \mathrm{d} \mu_{t}$ is absolutely continuous, a.e. differentiable, and

$$
\frac{d}{d t} \int_{\mathbb{R}^{d}} \zeta(x) \mathrm{d} \mu_{t}(x)=\int_{\mathbb{R}^{d}}\langle\boldsymbol{f}(t, x), \nabla \zeta(x)\rangle \mathrm{d} \mu_{t}(x)+\int_{\mathbb{R}^{d}}\left\langle\nabla \zeta(x), \mathrm{d} \boldsymbol{\nu}_{t}(x)\right\rangle \quad \text { for a.e. } t \in[0, T],
$$

for the vector field $\boldsymbol{f}(t, x):=\boldsymbol{F}\left(x, \mu_{t}\right)$ satisfying the structural bounds

$$
|\boldsymbol{f}(t, x)| \leq A+B\left(|x|+\int_{\mathbb{R}^{d}}|x| \mathrm{d} \mu_{t}\right) .
$$


In order to highlight the structural assumptions needed for the apriori estimates of this section, we introduce the set

$$
\begin{aligned}
\tilde{\mathscr{A}}:=\left\{(\mu, \boldsymbol{\nu}, \boldsymbol{f}): \mu \in A C\left([0, T] ; \mathcal{P}_{1}\left(\mathbb{R}^{d}\right)\right), \boldsymbol{\nu} \in \mathcal{M}\left([0, T] \times \mathbb{R}^{d} ; U\right), \mathcal{E}(\boldsymbol{\nu}, \tilde{\mu})<\infty,\right. \\
\left.f:[0, T] \times \mathbb{R}^{d} \rightarrow \mathbb{R}^{d} \text { Borel function satisfying (15.1) and (15.2) }\right\} ;
\end{aligned}
$$

the above discussion shows that if $(\mu, \boldsymbol{\nu}) \in \mathscr{A}$ then setting $\boldsymbol{f}(t, x):=\boldsymbol{F}\left(x, \mu_{t}\right)$ we have $(\mu, \boldsymbol{\nu}, \boldsymbol{f}) \in$ $\tilde{\mathscr{A}}$.

Firstly, let us show a uniform bound in time of the first moment, which is the infinite dimensional version of Lemma 4.1

Lemma 5.1 If $(\mu, \boldsymbol{\nu}, \boldsymbol{f}) \in \tilde{\mathscr{A}}$ then the following estimate holds true

$$
\sup _{t \in[0, T]} \int_{\mathbb{R}^{d}}|x| \mathrm{d} \mu_{t}(x) \leq\left(\int_{\mathbb{R}^{d}}|x| \mathrm{d} \mu_{0}(x)+A T+|\boldsymbol{\nu}|\left((0, T) \times \mathbb{R}^{d}\right)\right) e^{2 B T} .
$$

In particular, there exists a constant $M>0$ only depending on $A, B, T, \mathcal{E}(\mu, \boldsymbol{\nu})$ and $\int_{\mathbb{R}^{d}}|x| \mathrm{d} \mu_{0}$ such that

$$
|\boldsymbol{f}(t, x)| \leq M(1+|x|) \quad \text { for every }(t, x) \in[0, T] \times \mathbb{R}^{d} .
$$

Proof. Let $\zeta \in C_{c}^{1}\left(\mathbb{R}^{d}\right)$ be a cut-off function such that $0 \leq \zeta \leq 1$,

$$
\zeta(x)= \begin{cases}1 & \text { if }|x| \leq 1 \\ 0 & \text { if }|x| \geq 2\end{cases}
$$

and $|\nabla \zeta| \leq 1$. Let $\zeta_{n}$ be the sequence $\zeta_{n}(x):=\zeta(x / n)$. Consider now the product $\zeta_{n}(x)|x|$ and smooth it out in zero by substituting $|x|$ with $g_{\varepsilon}(x):=\sqrt{|x|^{2}+\varepsilon}$. Now $\zeta_{n} g_{\varepsilon}$ is a proper test function and the following equality holds true

$$
\begin{aligned}
& \int_{\mathbb{R}^{d}} \zeta_{n}(x) g_{\varepsilon}(x) \mathrm{d} \mu_{t}(x)-\int_{\mathbb{R}^{d}} \zeta_{n}(x) g_{\varepsilon}(x) \mathrm{d} \mu_{0}(x) \\
& \quad=\int_{0}^{t} \int_{\mathbb{R}^{d}}\left\langle\boldsymbol{f}(s, x), \nabla\left(\zeta_{n}(x) g_{\varepsilon}(x)\right)\right\rangle \mathrm{d} \mu_{s}(x) \mathrm{d} s+\int_{0}^{t} \int_{\mathbb{R}^{d}}\left\langle\nabla\left(\zeta_{n}(x) g_{\varepsilon}(x)\right), \mathrm{d} \boldsymbol{\nu}_{s}(x)\right\rangle \mathrm{d} s
\end{aligned}
$$

Thanks to

$$
\left|\nabla \zeta_{n}(x)\right| \leq \frac{1}{n}, \quad g_{\varepsilon}(x) \leq|x|+\sqrt{\varepsilon}, \quad\left|\nabla g_{\varepsilon}(x)\right|=\frac{|x|}{\sqrt{|x|^{2}+\varepsilon}} \leq 1
$$

we can write

$$
\begin{aligned}
& \int_{\mathbb{R}^{d}} \zeta_{n}(x) g_{\varepsilon}(x) d \mu_{t}(x)-\int_{\mathbb{R}^{d}} \zeta_{n}(x) g_{\varepsilon}(x) d \mu_{0}(x) \\
& \quad \leq\left(1+\frac{\sqrt{\varepsilon}}{n}\right) \int_{0}^{t} \int_{\mathbb{R}^{d}}|\boldsymbol{f}(s, x)| \mathrm{d} \mu_{s}(x) \mathrm{d} s+\left(1+\frac{\sqrt{\varepsilon}}{n}\right) \int_{0}^{t} \int_{\mathbb{R}^{d}} d\left|\boldsymbol{\nu}_{s}\right|(x) \mathrm{d} s .
\end{aligned}
$$

Apply now monotone convergence as $\varepsilon \rightarrow 0$ first, then let $n \rightarrow \infty$. Owing to $\zeta_{n}|x| \nearrow|x|$ we get

$$
\begin{gathered}
\int_{\mathbb{R}^{d}}|x| \mathrm{d} \mu_{t}(x)-\int_{\mathbb{R}^{d}}|x| \mathrm{d} \mu_{0}(x) \leq \int_{0}^{t} \int_{\mathbb{R}^{d}}|\boldsymbol{f}(s, x)| \mathrm{d} \mu_{s}(x) \mathrm{d} s+|\boldsymbol{\nu}|\left((0, T) \times \mathbb{R}^{d}\right) \\
\leq \int_{0}^{t} \int_{\mathbb{R}^{d}}\left[A+B\left(|x|+\int_{\mathbb{R}^{d}}|x| \mathrm{d} \mu_{s}(x)\right)\right] \mathrm{d} \mu_{s}(x) \mathrm{d} s+|\boldsymbol{\nu}|\left((0, T) \times \mathbb{R}^{d}\right) \\
\leq A T+2 B \int_{0}^{t} \int_{\mathbb{R}^{d}}|x| \mathrm{d} \mu_{s}(x) d s+|\boldsymbol{\nu}|\left((0, T) \times \mathbb{R}^{d}\right),
\end{gathered}
$$

and we conclude by Gronwall inequality. 
Lemma 5.2 If $(\mu, \boldsymbol{\nu}, \boldsymbol{f}) \in \tilde{\mathscr{A}}$ with $\boldsymbol{\nu}=\boldsymbol{v} \tilde{\mu}$, then for any $\vartheta \in C_{\mathrm{Lip}}^{1}\left(\mathbb{R}^{d}\right)$ the following equality holds

$$
\frac{d}{d t} \int_{\mathbb{R}^{d}} \vartheta(x) \mathrm{d} \mu_{t}(x)=\int_{\mathbb{R}^{d}}\langle\boldsymbol{f}(t, x)+\boldsymbol{v}(t, x), \nabla \vartheta(x)\rangle \mathrm{d} \mu_{t}(x) \quad \text { for a.e. } t \in[0, T],
$$

where $C_{\mathrm{Lip}}^{1}\left(\mathbb{R}^{d}\right)$ denotes the space of continuously differentiable functions with bounded gradient.

Proof. Let $\vartheta \in C_{\mathrm{Lip}}^{1}\left(\mathbb{R}^{d}\right)$ and $\zeta_{n}$ the sequence of cut-off functions defined in the proof of Lemma 5.1. Then $\zeta_{n} \vartheta$ is a test function and

$$
\begin{gathered}
\frac{d}{d t} \int_{\mathbb{R}^{d}} \zeta_{n}(x) \vartheta(x) \mathrm{d} \mu_{t}(x)=\int_{\mathbb{R}^{d}}\left\langle\boldsymbol{f}(t, x)+\boldsymbol{v}(t, x), \nabla\left(\zeta_{n}(x) \vartheta(x)\right)\right\rangle \mathrm{d} \mu_{t}(x) \\
\quad=\int_{\mathbb{R}^{d}}\left\langle\boldsymbol{f}(t, x)+\boldsymbol{v}(t, x), \nabla \zeta_{n}(x) \vartheta(x)+\zeta_{n}(x) \nabla \vartheta(x)\right\rangle \mathrm{d} \mu_{t}(x) .
\end{gathered}
$$

Taking into account that $\left|\nabla \zeta_{n}\right| \leq \frac{1}{n} \chi_{B_{2 n}}$, the Lipschitz continuity of $\vartheta$, the growth condition on $f$ and Lemma 5.1 by dominated convergence we obtain that

$$
\int_{\mathbb{R}^{d}} \vartheta(x) \mathrm{d} \mu_{t}(x)=\int_{\mathbb{R}^{d}} \vartheta(x) \mathrm{d} \mu_{0}(x)+\int_{\mathbb{R}^{d}}\langle\boldsymbol{f}(t, x)+\boldsymbol{v}(t, x), \nabla \vartheta(x)\rangle \mathrm{d} \mu_{t}(x) .
$$

Now we are ready to prove the main result of this section. It involves an auxiliary admissible function $\theta:[0, \infty) \rightarrow[0, \infty)$ (according to Definition 2.2) dominated by $\psi$, i.e.

$$
\theta(|x|) \leq 1+\psi(x) \text { for every } x \in U ;
$$

notice that, combining Lemma 2.3 and Remark 2.4 , if $\mu_{0} \in \mathcal{P}_{1}\left(\mathbb{R}^{d}\right)$ we can always find an admissible function $\theta$ satisfying (5.10) and

$$
\int_{\mathbb{R}^{d}} \theta(|x|) \mathrm{d} \mu_{0}(x)<\infty
$$

Proposition 5.3 Let $(\mu, \boldsymbol{\nu}, \boldsymbol{f}) \in \tilde{\mathscr{A}}$ and let $\theta$ be an admissible function satisfying (5.10) and (5.11). Then there exists a constant $C>0$, depending only on $A, B, T, \int_{\mathbb{R}^{d}}|x| \mathrm{d} \mu_{0}(x), \mathcal{E}(\mu, \boldsymbol{\nu})$, $\theta(1)$ and the doubling constant $K$ of $\theta$ (see (2.9)), such that

$$
\sup _{t \in[0, T]} \int_{\mathbb{R}^{d}} \theta(|x|) \mathrm{d} \mu_{t}(x) \leq C\left(1+\int_{\mathbb{R}^{d}} \theta(|x|) \mathrm{d} \mu_{0}(x)\right) .
$$

Proof. Since $\mathcal{E}(\mu, \boldsymbol{\nu})<+\infty$, we have that $\boldsymbol{\nu}=\boldsymbol{v} \tilde{\mu}$. We also set $\vartheta(x):=\theta(|x|), x \in \mathbb{R}^{d}$.

STEP 1: We start by approximating $\theta$ from below with a sequence of $C^{1}$-Lipschitz functions

$$
\begin{gathered}
\vartheta^{n}(x):=\theta^{n}(|x|), \quad \theta^{n}(r):= \begin{cases}\theta(r) & \text { if }|x| \leq n \\
\theta^{\prime}(n)(r-n)+\theta(n) & \text { if } r>n .\end{cases} \\
\int_{\mathbb{R}^{d}} \vartheta^{n}(x) \mathrm{d} \mu_{t}(x)=\int_{\mathbb{R}^{d}} \vartheta^{n}(x) \mathrm{d} \mu_{0}(x)+\int_{0}^{t} \int_{\mathbb{R}^{d}}\left\langle\boldsymbol{f}(s, x)+\boldsymbol{v}(s, x), \nabla \vartheta^{n}(x)\right\rangle \mathrm{d} \mu_{s}(x) \mathrm{d} s \\
\leq \int_{\mathbb{R}^{d}} \vartheta^{n}(x) \mathrm{d} \mu_{0}(x)+\int_{0}^{t} \int_{\mathbb{R}^{d}}|\boldsymbol{f}(s, x)+\boldsymbol{v}(s, x)|\left|\nabla \vartheta^{n}(x)\right| \mathrm{d} \mu_{s}(x) \mathrm{d} s .
\end{gathered}
$$

By construction, $\theta^{n}(|x|) \nearrow \theta(|x|)\left|\nabla \vartheta^{n}(x)\right| \nearrow|\nabla \vartheta(x)|$, for every $x \in \mathbb{R}^{d}$; we can thus pass to the limit in the relation above to get

$$
\int_{\mathbb{R}^{d}} \vartheta(x) \mathrm{d} \mu_{t}(x) \leq \int_{\mathbb{R}^{d}} \vartheta(x) \mathrm{d} \mu_{0}(x)+\int_{0}^{t} \int_{\mathbb{R}^{d}}|\boldsymbol{f}(s, x)+\boldsymbol{v}(s, x) \| \nabla \vartheta(x)| \mathrm{d} \mu_{s}(x) \mathrm{d} s .
$$


STEP 2: We want to estimate the right hand side of (5.15). Since $\theta^{\prime}(r) \geq 0$ by (2.11) and $|\nabla \vartheta(x)|=\left|\theta^{\prime}(|x|) \frac{x}{|x|}\right|=\theta^{\prime}(|x|)$, so that (15.5) yields

$$
\int_{\mathbb{R}^{d}}|\boldsymbol{f}(s, x)||\nabla \vartheta(x)| \mathrm{d} \mu_{s}(x) \leq M \int_{\mathbb{R}^{d}}(1+|x|) \theta^{\prime}(|x|) \mathrm{d} \mu_{s}(x)
$$

By the monotonicity of $\theta^{\prime}(2.15)$ in $[0,1]$ and (2.11), we have

$$
(1+r) \theta^{\prime}(r) \leq 2 K(1+\theta(1)+\theta(r))
$$

so that

$$
\int_{\mathbb{R}^{d}}|\boldsymbol{f}(s, x)||\nabla \vartheta(x)| \mathrm{d} \mu_{s}(x) \leq 2 M K\left(1+\theta(1)+\int_{\mathbb{R}^{d}} \theta(|x|) \mathrm{d} \mu_{s}(x)\right) .
$$

Concerning the second term on the right hand side of (5.15)

$$
\begin{aligned}
|\boldsymbol{v}(s, x)||\nabla \vartheta(x)| & \leq \theta(|\boldsymbol{v}(s, x)|)+\theta^{*}(|\nabla \vartheta(x)|) \\
& =\theta(|\boldsymbol{v}(s, x)|)+\theta^{*}\left(\theta^{\prime}(|x|)\right) \\
& =\theta(|\boldsymbol{v}(s, x)|)+\theta^{\prime}(|x|)|x|-\theta(|x|) \\
& \leq \theta(|\boldsymbol{v}(s, x)|)+K(1+\theta(|x|)),
\end{aligned}
$$

where the equality $\theta(|x|)+\theta^{*}\left(\theta^{\prime}(|x|)\right)=\theta^{\prime}(|x|)|x|$ comes from the definition of the Fenchel conjugate $\theta^{*}$. What we end up with is the following

$$
\begin{aligned}
\int_{0}^{t} \int_{\mathbb{R}^{d}} & |\boldsymbol{v}(s, x)||\nabla \vartheta(x)| \mathrm{d} \mu_{s}(x) \mathrm{d} s \\
& \leq \int_{0}^{T} \int_{\mathbb{R}^{d}} \theta(|\boldsymbol{v}(s, x)|) \mathrm{d} \mu_{s}(x) \mathrm{d} s+K t+K \int_{0}^{t} \int_{\mathbb{R}^{d}} \theta(|x|) \mathrm{d} \mu_{s}(x) \mathrm{d} s \\
& \leq T \mathcal{E}(\mu, \boldsymbol{\nu})+(1+K) T+K \int_{0}^{t} \int_{\mathbb{R}^{d}} \theta(|x|) \mathrm{d} \mu_{s}(x) \mathrm{d} s
\end{aligned}
$$

Summing up the two estimates we obtain for every $t \in[0, T]$ and a suitable constant $C>0$

$$
\int_{\mathbb{R}^{d}} \theta(|x|) \mathrm{d} \mu_{t}(x) \leq \int_{\mathbb{R}^{d}} \theta(|x|) \mathrm{d} \mu_{0}(x)+C T+C \int_{0}^{t} \int_{\mathbb{R}^{d}} \theta(|x|) \mathrm{d} \mu_{s}(x) \mathrm{d} s,
$$

and thanks to the Gronwall inequality we get

$$
\int_{\mathbb{R}^{d}} \theta(|x|) \mathrm{d} \mu_{t}(x) \leq e^{C T}\left(\int_{\mathbb{R}^{d}} \theta(|x|) \mathrm{d} \mu_{0}(x)+C T\right) .
$$

\section{Proof of the main Theorems.}

\subsection{The superposition principle}

We first recall the superposition principle for solutions of the continuity equation

$$
\partial_{t} \mu_{t}+\nabla \cdot\left(\boldsymbol{w}(t, \cdot) \mu_{t}\right)=0 .
$$

Let us denote with $\Gamma_{T}$ the complete and separable metric space of continuous functions from $[0, T]$ to $\mathbb{R}^{d}$ endowed with the sup-distance and introduce the evaluation maps $e_{t}: \Gamma_{T} \rightarrow \mathbb{R}^{d}$ defined by $e_{t}(\gamma):=\gamma(t)$, for $t \in[0, T]$. The following result holds 
Theorem 6.1 (Superposition principle) Let $\mu_{t}$ be a narrowly continuous weak solution to (6.1) with a velocity field $\boldsymbol{w}$ satisfying

$$
\int_{0}^{T} \int_{\mathbb{R}^{d}}|\boldsymbol{w}(t, x)| \mathrm{d} \mu_{t}(x) \mathrm{d} t<+\infty .
$$

Then there exists $\pi \in \mathcal{P}\left(\Gamma_{T}\right)$ concentrated on the set of curves $\gamma \in A C\left([0, T] ; \mathbb{R}^{d}\right)$ such that

$$
\dot{\gamma}(t)=\boldsymbol{w}(t, \gamma(t)) \quad \text { for a.e. } t \in[0, T] .
$$

Moreover, $\mu_{t}=\left(e_{t}\right)_{\#} \pi$ for any $t \in[0, T]$, i.e.

$$
\int_{\mathbb{R}^{d}} \vartheta(y) \mathrm{d} \mu_{t}(y)=\int_{\Gamma_{T}} \vartheta(\gamma(t)) \mathrm{d} \pi(\gamma), \quad \forall \vartheta \in C_{b}\left(\mathbb{R}^{d}\right) .
$$

For the proof we refer to $[3,3.4]$.

\section{$6.2 \Gamma$-convergence.}

Let us start with a preliminary lemma.

Lemma 6.2 Let $(\boldsymbol{x}, \boldsymbol{u}) \in A C\left([0, T] ;\left(\mathbb{R}^{d}\right)^{N}\right) \times L^{1}\left([0, T] ; U^{N}\right)$ and $\mu=\mu[\boldsymbol{x}], \boldsymbol{\nu}=\boldsymbol{\nu}[\boldsymbol{x}, \boldsymbol{u}]$. Then we have

$$
\frac{1}{N} \sum_{i=1}^{N} \psi\left(u_{i}(t)\right) \geq \Psi\left(\boldsymbol{\nu}_{t} \mid \mu_{t}\right) \quad \text { for a.e. } t \in[0, T] .
$$

Moreover, if $(\boldsymbol{x}, \boldsymbol{u}) \in \mathscr{A}^{N}$ then

$$
\frac{1}{N} \sum_{i=1}^{N} \psi\left(u_{i}(t)\right)=\Psi\left(\boldsymbol{\nu}_{t} \mid \mu_{t}\right) \quad \text { for a.e. } t \in[0, T] .
$$

Proof. Let us first compute the density of $\boldsymbol{\nu}$ w.r.t. $\tilde{\mu}$. We introduce the finite set $I_{N}:=$ $\{1,2, \cdots, N\}$ with the discrete topology and the normalized counting measure $\sigma_{N}=\frac{1}{N} \sum_{i=1}^{N} \delta_{i}$. We can identify $\boldsymbol{x}$ with a continuous map from $[0, T] \times I_{N}$ to $\mathbb{R}^{d}, \boldsymbol{x}(t, i):=x_{i}(t)$, so that $\mu_{t}=\boldsymbol{x}(t, \cdot)_{\sharp} \sigma_{N}$. Similarly, we set $\boldsymbol{u}(t, i):=u_{i}(t)$, where $\boldsymbol{u}:[0, T] \rightarrow U^{N}$ is a Borel representative. In order to represent $\tilde{\mu}$ and $\boldsymbol{\nu}$ it is useful to deal with the map $\boldsymbol{y}:[0, T] \times I_{N} \rightarrow[0, T] \times \mathbb{R}^{d}$, $\boldsymbol{y}(t, i):=(t, \boldsymbol{x}(t, i))$, which yields $\tilde{\mu}=\boldsymbol{y}_{\sharp}\left(\lambda \otimes \sigma_{N}\right)$ and $\boldsymbol{\nu}=\boldsymbol{y}_{\sharp}\left(\boldsymbol{u} \cdot\left(\lambda \otimes \sigma_{N}\right)\right)$. We denote by $Y \subset[0, T] \times \mathbb{R}^{d}$ the range of $\boldsymbol{y}$, and by

$$
X(t)=\left\{x \in \mathbb{R}^{d}:(t, x) \in Y\right\}=\left\{x \in \mathbb{R}^{d}: x_{i}(t)=x \text { for some } i \in I_{N}\right\}
$$

its fibers. For every $(t, x) \in[0, T] \times \mathbb{R}^{d}$ we will also consider the set

$$
J(t, x):=\left\{i \in I_{N}: x_{i}(t)=x\right\} \text { with its characteristic function } \quad \chi_{t, x}(i):= \begin{cases}1 & \text { if } x_{i}(t)=x \\ 0 & \text { otherwise }\end{cases}
$$

For every $t \in[0, T]$ the collection $\left\{\chi_{t, x}: x \in X(t)\right\}$ provides a partition of unity of $I_{N}$ and for every $i \in I_{N}$ the map $(t, x) \mapsto \chi_{t, x}$ is upper semicontinuous in $[0, T] \times \mathbb{R}^{d}$. The conditional measures $\tilde{\mu}_{t, x} \in \mathcal{P}\left(I_{N}\right)$ are then defined by

$$
\tilde{\mu}_{t, x}(J):=\sigma_{N}(J \cap J(t, x)) / \sigma_{N}(J(t, x)), \quad(t, x) \in Y ;
$$

since for every $J \subset I_{N}$

$$
\sigma_{N}(J \cap J(t, x))=\int_{J} \chi_{t, x} \mathrm{~d} \sigma_{N}=\frac{1}{N} \sum_{i \in J} \chi_{t, x}(i),
$$


the map $(t, x) \mapsto \sigma_{N}(J \cap J(t, x))$ is also upper semicontinuous and $\tilde{\mu}_{t, x}$ is a Borel family.

One immediately checks that $\tilde{\mu}_{t, x}$ provides a disintegration (see e.g. [5. Theorem 5.3.1]) of $\lambda \otimes \sigma_{N}$ w.r.t. the map $\boldsymbol{y}$, i.e.

$$
\lambda \otimes \sigma_{N}=\int \tilde{\mu}_{t, x} \mathrm{~d} \tilde{\mu}(t, x) .
$$

Since $\boldsymbol{\nu}=\boldsymbol{y}_{\sharp}\left(\boldsymbol{u} \cdot\left(\lambda \otimes \sigma_{N}\right)\right)$, we eventually end up with the representation formula for the Borel vector field $\boldsymbol{v}$

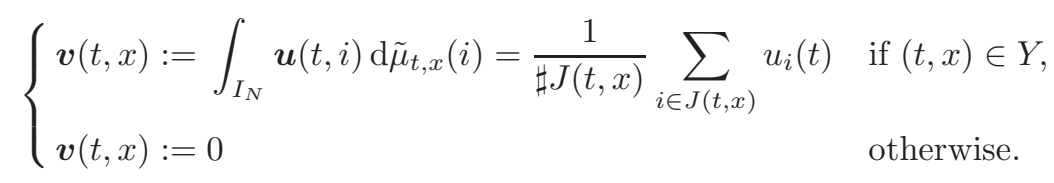

In particular

$$
\boldsymbol{\nu}_{t}=\boldsymbol{v}(t, \cdot) \mu_{t}, \quad \mu_{t}=\sum_{x \in X(t)} \frac{\sharp J(t, x)}{N} \delta_{x}
$$

and consequently

$$
\Psi\left(\boldsymbol{\nu}_{t} \mid \mu_{t}\right)=\int_{\mathbb{R}^{d}} \psi(\boldsymbol{v}(t, x)) \mathrm{d} \mu_{t}(x)=\sum_{x \in X(t)} \frac{\sharp J(t, x)}{N} \psi\left(\frac{1}{\sharp J(t, x)} \sum_{i \in J(t, x)} u_{i}(t)\right) .
$$

The convexity of $\psi$ immediately yields

$$
\Psi\left(\boldsymbol{\nu}_{t} \mid \mu_{t}\right) \leq \frac{1}{N} \sum_{i=1}^{N} \psi\left(u_{i}(t)\right)
$$

Let us show that equality holds in (6.12) if $(\boldsymbol{x}, \boldsymbol{u}) \in \mathscr{A}^{N}$.

Let $\mathscr{P}$ be the collection of all the partitions $P$ of $I_{N}$. It is clear that for every $t \in[0, T]$ the family $P_{\boldsymbol{x}}(t):=\{J(t, x): x \in X(t)\}$ is an element of $\mathscr{P}$; moreover for every $P \in \mathscr{P}$ the set

$$
S_{P}:=\left\{t \in[0, T]: P_{\boldsymbol{x}}(t)=P\right\} \quad \text { is Borel. }
$$

To show (6.13) we introduce an order relation on $\mathscr{P}$ : we say that $P_{1} \prec P_{2}$ if every element of $P_{1}$ is contained in some element of $P_{2}$. We denote by $\hat{P}:=\{Q \in \mathscr{P}: P \prec Q\}$ the collection of all the partitions $Q$ coarser than $P$.

It is easy to check that for every $P \in \mathscr{P}$ the set $P_{\boldsymbol{x}}^{-1}(\hat{P})=\left\{t \in[0, T]: P_{\boldsymbol{x}}(t) \in \hat{P}\right\}$ is closed. In fact, if $P_{\boldsymbol{x}}(t) \notin \hat{P}$ then there is a set $I \in P$ not contained in any element of $P_{\boldsymbol{x}}(t)$, so that we can find two indices $i, j \in I$ belonging to different elements of $P_{\boldsymbol{x}}(t)$, i.e. $x_{i}(t) \neq x_{j}(t)$. By continuity, this relation holds in a neighborhood $U$ of $t$, so that $P_{\boldsymbol{x}}(s) \notin \hat{P}$ for every $s \in U$.

Since for every partition $P \in \mathscr{P}\{P\}=\hat{P} \backslash \cup\{\hat{Q}: Q \in \hat{P}, Q \neq P\}$, it follows that

$$
S_{P}=P_{\boldsymbol{x}}^{-1}(\hat{P}) \backslash \bigcup_{Q \in \hat{P}, Q \neq P} P_{\boldsymbol{x}}^{-1}(\hat{Q}),
$$

so that $S_{P}$ is the difference between closed sets and (6.13) holds.

We can therefore decompose the interval $[0, T]$ in the finite Borel partition $\left\{S_{P}: P \in \mathscr{P}\right\}$. On the other hand, for every partition $P \in \mathscr{P}$ and every pair of indices $i, j$ in $I \in P$ we have $x_{i}(t)=x_{j}(t)$ in $S_{P}$ so that $\dot{x}_{i}(t)=\dot{x}_{j}(t)$ for $\lambda$-almost every $t \in S_{P}$ and consequently, by (3.9), we obtain that $u_{i}(t)=u_{j}(t)$ for $\lambda$-a.e. $t \in S_{P}$. We eventually deduce

$$
\sharp I \psi\left(\frac{1}{\sharp I} \sum_{i \in I} u_{i}(t)\right)=\sum_{i \in I} \psi\left(u_{i}(t)\right) \quad \text { for every } I \in P_{\boldsymbol{x}}(t), \quad \lambda \text {-a.e. in } S_{P},
$$


and therefore, by (6.11),

$$
\begin{aligned}
\Psi\left(\boldsymbol{\nu}_{t} \mid \mu_{t}\right) & =\sum_{I \in P_{\boldsymbol{x}}(t)} \frac{\sharp I}{N} \psi\left(\frac{1}{\sharp I} \sum_{i \in I} u_{i}(t)\right)=\frac{1}{N} \sum_{I \in P_{\boldsymbol{x}}(t)} \sum_{i \in I} \psi\left(u_{i}(t)\right) \\
& =\sum_{i=1}^{N} \psi\left(u_{i}(t)\right) \quad \text { for } \lambda \text {-a.e. } t \in S_{P} .
\end{aligned}
$$

Since $\left\{S_{P}: P \in \mathscr{P}\right\}$ is a finite Borel partition of $[0, T]$, we get [6.6).

Proof. of Theorem 3.2. The liminf inequality.

Let $(\mu, \boldsymbol{\nu}) \in A C\left([0, T] ; \mathcal{P}_{1}\left(\mathbb{R}^{d}\right)\right) \times \mathcal{M}\left([0, T] \times \mathbb{R}^{d} ; U\right)$ and $\left(\boldsymbol{x}^{N}, \boldsymbol{u}^{N}\right) \in A C\left([0, T] ;\left(\mathbb{R}^{d}\right)^{N}\right) \times$ $L^{1}\left([0, T] ; U^{N}\right), N \in \mathbb{N}$, such that $\mu^{N}=\mu\left[\boldsymbol{x}^{N}\right] \rightarrow \mu$ in $C\left([0, T] ; \mathcal{P}_{1}\left(\mathbb{R}^{d}\right)\right)$ and $\boldsymbol{\nu}^{N}=\boldsymbol{\nu}\left[\boldsymbol{x}^{N}, \boldsymbol{u}^{N}\right] \boldsymbol{十}^{*} \boldsymbol{\nu}$ in $\mathcal{M}\left([0, T] \times \mathbb{R}^{d} ; U\right)$.

Since $L^{N} \geq 0, L^{N}\left(x, \boldsymbol{x}^{N}(t)\right) \rightarrow L\left(x, \mu_{t}\right)$ on compact sets, and $\mu\left[\boldsymbol{x}^{N}\right] \rightarrow^{*} \mu$, then for every compact $K \subset \mathbb{R}^{d}$ by (3.1) we have

$$
\begin{aligned}
\liminf _{N \rightarrow+\infty} & \int_{\mathbb{R}^{d}} L^{N}\left(x, \boldsymbol{x}^{N}(t)\right) \mathrm{d} \mu_{t}^{N}(x) \\
& \geq \liminf _{N \rightarrow+\infty} \int_{K} L^{N}\left(x, \boldsymbol{x}^{N}(t)\right) \mathrm{d} \mu_{t}^{n}(x)=\int_{K} L\left(x, \mu_{t}\right) \mathrm{d} \mu_{t}(x) .
\end{aligned}
$$

Since

$$
\frac{1}{N} \sum_{i=1}^{N} L^{N}\left(x_{i}^{N}(t), \boldsymbol{x}^{N}(t)\right)=\int_{\mathbb{R}^{d}} L^{N}\left(x, \boldsymbol{x}^{N}(t)\right) \mathrm{d} \mu_{t}^{N}(x)
$$

and $L \geq 0$, by (6.15) we obtain

$$
\liminf _{N \rightarrow \infty} f_{0}^{T} \frac{1}{N} \sum_{i=1}^{N} L^{N}\left(x_{i}^{N}(t), \boldsymbol{x}^{N}(t)\right) \mathrm{d} t \geq f_{0}^{T} \int_{\mathbb{R}^{d}} L\left(x, \mu_{t}\right) \mathrm{d} \mu_{t}(x) \mathrm{d} t .
$$

By (6.5) we have

$$
\frac{1}{N} \sum_{i=1}^{N} \psi\left(u_{i}^{N}(t)\right) \geq \Psi\left(\boldsymbol{\nu}_{t}^{N} \mid \mu_{t}^{N}\right) \quad \text { for a.e. } t \in[0, T] .
$$

and Theorem 2.6 yields

$$
\liminf _{N \rightarrow \infty} f_{0}^{T} \Psi\left(\boldsymbol{\nu}_{t}^{N} \mid \mu_{t}^{N}\right) \mathrm{d} t=\liminf _{N \rightarrow \infty} \Psi\left(\boldsymbol{\nu}^{N} \mid \tilde{\mu}^{N}\right) \geq \Psi(\boldsymbol{\nu} \mid \tilde{\mu})=f_{0}^{T} \Psi\left(\boldsymbol{\nu}_{t} \mid \mu_{t}\right) \mathrm{d} t .
$$

By (6.16), (6.17), and (6.18) it follows (3.16).

The limsup inequality. Recall that $\phi$ is an admissible function satisfying (2.10). Let $(\mu, \boldsymbol{\nu}) \in \mathscr{A}$ such that $\mathcal{E}(\mu, \boldsymbol{\nu})<+\infty$ and $\int_{\mathbb{R}^{d}} \phi(|x|) \mathrm{d} \mu_{0}(x)<\infty$.

Since $\Psi(\boldsymbol{\nu} \mid \tilde{\mu})<+\infty$ we have $\boldsymbol{\nu}=\boldsymbol{v} \tilde{\mu}$ for a Borel vector field $\boldsymbol{v}:[0, T] \times \mathbb{R}^{d} \rightarrow U$. Since $(\mu, \nu) \in \mathscr{A}$ the continuity equation

$$
\partial_{t} \mu_{t}+\nabla \cdot\left(\boldsymbol{w}(t, \cdot) \mu_{t}\right)=0
$$

holds with the vector field $\boldsymbol{w}(t, x):=\boldsymbol{f}(t, x)+\boldsymbol{v}(t, x), \boldsymbol{f}(t, x):=\boldsymbol{F}\left(x, \mu_{t}\right)$. By (3.6) and Lemma 5.1 we have that

$$
\boldsymbol{f} \in C\left([0, T] \times \mathbb{R}^{d}\right), \quad|\boldsymbol{f}(t, x)| \leq M(1+|x|), \quad \int_{0}^{T} \int_{\mathbb{R}^{d}}|\boldsymbol{w}(t, x)| \mathrm{d} \mu_{t}(x) \mathrm{d} t<+\infty .
$$


By Theorem 6.1 there exists a probability measure $\pi \in \mathcal{P}\left(\Gamma_{T}\right)$ such that $\left(e_{t}\right)_{\#} \pi=\mu_{t}$ for every $t \in[0, T]$ and it is concentrated on the absolutely continuous solutions of the ODE

$$
\dot{\gamma}(t)=\boldsymbol{f}(t, \gamma(t))+\boldsymbol{v}(t, \gamma(t)) .
$$

The strategy of the proof consists in finding an appropriate sequence of measures $\pi^{N} \in \mathcal{P}^{N}\left(\Gamma_{T}\right)$ narrowly convergent to $\pi$, defining $\mu_{t}^{N}:=\left(e_{t}\right)_{\sharp} \pi^{N}$ and $\boldsymbol{x}^{N}$ a corresponding curve such that $\mu\left[\boldsymbol{x}^{N}\right]=$ $\mu^{N}$. Then the objective is to construct a suitable sequence of controls $\boldsymbol{u}^{N}$ in such a way that the sequence $\left(\boldsymbol{x}^{N}, \boldsymbol{u}^{N}\right)$ belongs to $\mathscr{A}^{N}, \mu^{N} \rightarrow \mu$ in $C\left([0, T] ; \mathcal{P}_{1}\left(\mathbb{R}^{d}\right)\right), \boldsymbol{\nu}^{N}=\boldsymbol{\nu}\left[\boldsymbol{x}^{N}, \boldsymbol{u}^{N}\right] \rightarrow^{*} \boldsymbol{\nu}$ in $\mathcal{M}\left([0, T] \times \mathbb{R}^{d} ; U\right)$ and 3.20 holds.

STEP 1: (Definition of auxiliary functionals.)

We define the set

$$
A:=\left\{\gamma \in \Gamma_{T}: \gamma \in A C\left([0, T] ; \mathbb{R}^{d}\right), \text { (6.21) holds for a.e. } t \in[0, T]\right\}
$$

and we observe that $\pi(A)=1$.

Starting from $\mu$ and $L$ we define the functional $\mathcal{L}: A \rightarrow[0,+\infty)$ by

$$
\mathcal{L}(\gamma):=f_{0}^{T} L\left(\gamma(t), \mu_{t}\right) \mathrm{d} t
$$

Starting from $\psi$ and $\boldsymbol{v}$ we define the functional $\mathcal{F}: A \rightarrow[0,+\infty)$ by

$$
\mathcal{F}(\gamma):=f_{0}^{T} \psi(\boldsymbol{v}(t, \gamma(t))) \mathrm{d} t .
$$

By Fubini's theorem and the finiteness of $\mathcal{E}(\mu, \boldsymbol{\nu})$ we have

$$
f_{0}^{T} \int_{\mathbb{R}^{d}} L\left(x, \mu_{t}\right) \mathrm{d} \mu_{t}(x) \mathrm{d} t=f_{0}^{T} \int_{A} L\left(e_{t}(\gamma), \mu_{t}\right) \mathrm{d} \pi(\gamma) \mathrm{d} t=\int_{A} \mathcal{L}(\gamma) \mathrm{d} \pi(\gamma)
$$

and

$$
f_{0}^{T} \int_{\mathbb{R}^{d}} \psi(\boldsymbol{v}(t, x)) \mathrm{d} \mu_{t}(x) \mathrm{d} t=f_{0}^{T} \int_{A} \psi\left(\boldsymbol{v}\left(t, e_{t}(\gamma)\right) \mathrm{d} \pi(\gamma) \mathrm{d} t=\int_{A} \mathcal{F}(\gamma) \mathrm{d} \pi(\gamma)\right.
$$

We define the functional $\mathcal{H}: A \rightarrow[0,+\infty)$ by

$$
\mathcal{H}(\gamma):=f_{0}^{T} \phi(\mid \boldsymbol{v}(t, \gamma(t) \mid) \mathrm{d} t
$$

Starting by $\phi$ satisfying (2.10) we define the functional $\mathcal{G}: A \rightarrow[0,+\infty)$ by

$$
\mathcal{G}(\gamma):=\phi(|\gamma(0)|)+f_{0}^{T} \phi(|\gamma(t)|) \mathrm{d} t .
$$

It is not difficult to show that $\mathcal{G}$ and $\mathcal{L}$ are continuous. Here we prove that $\mathcal{F}$ and $\mathcal{H}$ are lowersemicontinuous. Let $\gamma \in A$ and $\left(\gamma_{k}\right)_{k \in \mathbb{N}}$ be a sequence in $A$ such that $\lim _{k \rightarrow+\infty} \sup _{t \in[0, T]} \mid \gamma_{k}(t)-$ $\gamma(t) \mid=0$. We define the sequence $f_{k} \in L^{1}\left([0, T] ; \mathbb{R}^{d}\right)$ by $f_{k}(t):=\boldsymbol{v}\left(t, \gamma_{k}(t)\right)$.

If $\sup _{k \in \mathbb{N}} f_{0}^{T} \phi\left(\mid \boldsymbol{v}\left(t, \gamma_{k}(t) \mid\right) \mathrm{d} t<+\infty\right.$, then by de la Vallée Poussin's criterion 4, Proposition 1.12] for equi-integrability and Dunford-Pettis theorem there exist $g \in L^{1}\left([0, T] ; \mathbb{R}^{d}\right)$ and a subsequence (not relabeled) of $f_{k}$ weakly convergent in $L^{1}\left([0, T] ; \mathbb{R}^{d}\right)$ to $g$ such that

$$
\liminf _{k \in \mathbb{N}} f_{0}^{T} \phi\left(\mid \boldsymbol{v}\left(t, \gamma_{k}(t) \mid\right) \mathrm{d} t \geq f_{0}^{T} \phi(|g(t)|) \mathrm{d} t .\right.
$$


Since $\gamma_{k}$ satisfies

$$
\gamma_{k}\left(t_{2}\right)-\gamma_{k}\left(t_{1}\right)=\int_{t_{1}}^{t_{2}}\left[\boldsymbol{v}\left(t, \gamma_{k}(t)\right)+\boldsymbol{f}\left(t, \gamma_{k}(t)\right)\right] \mathrm{d} t, \quad \forall t_{1}, t_{2} \in[0, T]
$$

and $\gamma$ satisfies

$$
\gamma\left(t_{2}\right)-\gamma\left(t_{1}\right)=\int_{t_{1}}^{t_{2}}[\boldsymbol{v}(t, \gamma(t))+\boldsymbol{f}(t, \gamma(t))] \mathrm{d} t, \quad \forall t_{1}, t_{2} \in[0, T]
$$

passing to the limit in (6.24) as $k \rightarrow \infty$ we obtain

$$
\gamma\left(t_{2}\right)-\gamma\left(t_{1}\right)=\int_{t_{1}}^{t_{2}}[g(t)+\boldsymbol{f}(t, \gamma(t))] \mathrm{d} t .
$$

By (6.25) it holds

$$
\int_{t_{1}}^{t_{2}} g(t) \mathrm{d} t=\int_{t_{1}}^{t_{2}} \boldsymbol{v}(t, \gamma(t)) \mathrm{d} t, \quad \forall t_{1}, t_{2} \in[0, T]
$$

and Lebesgue differentiation Theorem yields $g(t)=\boldsymbol{v}(t, \gamma(t))$ for a.e. $t \in[0, T]$.

STEP 2: (Construction of $\pi^{N}$.) We define the function $\mathfrak{F}:=(\mathcal{F}, \mathcal{L}, \mathcal{G}, \mathcal{H}): A \rightarrow \mathbb{R}^{4}$.

Notice that the finiteness of $\mathcal{E}(\mu, \boldsymbol{\nu})$, (6.22), and (6.23) imply that $\int_{A} \mathcal{F}(\gamma) \mathrm{d} \pi(\gamma)<+\infty$, $\int_{A} \mathcal{L}(\gamma) \mathrm{d} \pi(\gamma)<+\infty$ and $\int_{A} \mathcal{H}(\gamma) \mathrm{d} \pi(\gamma)<+\infty$. Since $\int_{A} \mathcal{G}(\gamma) \mathrm{d} \pi(\gamma)=\int_{0}^{T} \int_{\mathbb{R}^{d}} \phi(|x|) \mathrm{d} \mu_{t}(x) \mathrm{d} t$, by Proposition 5.3 we also have that $\int_{A} \mathcal{G}(\gamma) \mathrm{d} \pi(\gamma)<+\infty$.

By Lusin's theorem applied to the space $A$ with the measure $\pi$ and the function $\mathfrak{F}$ there exists a sequence of compact sets $A_{k}$ such that $A_{k} \subset A_{k+1} \subset A, \pi\left(A \backslash A_{k}\right)<\frac{1}{k}$, for all $k \geq 1$, and $\mathfrak{F}_{\mid A_{k}}$ is continuous. Moreover we have

$$
\lim _{k \rightarrow \infty} \pi\left(A_{k}\right)=\pi\left(\bigcup_{j=1}^{\infty} A_{j}\right)=1, \quad \pi\left(A \backslash \bigcup_{j=1}^{\infty} A_{j}\right)=0 .
$$

Then we define $\tilde{\pi}^{k} \in \mathcal{P}\left(\Gamma_{T}\right)$ by

$$
\tilde{\pi}^{k}:=\frac{1}{\pi\left(A_{k}\right)} \pi\left\llcorner A_{k} .\right.
$$

It is easy to check that $\left(\tilde{\pi}^{k}\right)_{k \in \mathbb{N}}$ weakly converges to $\pi$ as $k \rightarrow \infty$; since for each component $\mathfrak{F}_{j}$, $j=1,2,3,4$, of $\mathfrak{F}$ is nonnegative, Beppo Levi monotone convergence Theorem yields

$$
\lim _{k \rightarrow+\infty} \int_{A_{k}} \mathfrak{F}_{j}(\gamma) \mathrm{d} \pi(\gamma)=\int_{\bigcup_{k=1}^{\infty} A_{k}} \mathfrak{F}_{j}(\gamma) \mathrm{d} \pi(\gamma)=\int_{A} \mathfrak{F}_{j}(\gamma) \mathrm{d} \pi(\gamma),
$$

and (6.28) easily yields

$$
\lim _{k \rightarrow \infty}\left|\int_{\Gamma_{T}} \mathfrak{F}(\gamma) \mathrm{d} \tilde{\pi}^{k}(\gamma)-\int_{\Gamma_{T}} \mathfrak{F}(\gamma) \mathrm{d} \pi(\gamma)\right|=0
$$

Since $A_{k}$ is compact, we can find a sequence of atomic measures

$$
m \mapsto \tilde{\pi}_{m}^{k}:=\frac{1}{m} \sum_{i=1}^{m} \delta_{\gamma_{i, k, m}}, \quad \gamma_{i, k, m} \in A_{k},
$$

narrowly convergent to $\tilde{\pi}^{k}$ as $m \rightarrow+\infty$. Since $\mathfrak{F}_{\mid A_{k}}$ is bounded and continuous, in particular it holds that

$$
\lim _{m \rightarrow \infty} \int_{\Gamma_{T}} \mathfrak{F}(\gamma) \mathrm{d} \tilde{\pi}_{m}^{k}(\gamma)=\int_{\Gamma_{T}} \mathfrak{F}(\gamma) \mathrm{d} \tilde{\pi}^{k}(\gamma)
$$


Hence, for every $k \in \mathbb{N}$ there exists $\bar{m}(k)$ satisfying

$$
W\left(\tilde{\pi}_{m}^{k}, \tilde{\pi}^{k}\right) \leq \frac{1}{k} \quad \text { and } \quad\left|\int_{\Gamma_{T}} \mathfrak{F}(\gamma) \mathrm{d} \tilde{\pi}_{m}^{k}(\gamma)-\int_{\Gamma_{T}} \mathfrak{F}(\gamma) \mathrm{d} \tilde{\pi}^{k}(\gamma)\right| \leq \frac{1}{k}, \quad \forall m \geq \bar{m}(k),
$$

where $W$ is any distance metrizing the weak convergence.

We define $\bar{\pi}^{k}:=\tilde{\pi}_{\bar{m}(k)}^{k}$ and we clearly have that $\bar{\pi}^{k} \in \mathcal{P}^{\bar{m}(k)}\left(\Gamma_{T}\right), W\left(\bar{\pi}^{k}, \pi\right) \rightarrow 0$ as $k \rightarrow \infty$ and, by (6.31) and (6.33),

$$
\lim _{k \rightarrow \infty}\left|\int_{\Gamma_{T}} \mathfrak{F}(\gamma) \mathrm{d} \bar{\pi}^{k}(\gamma)-\int_{\Gamma_{T}} \mathfrak{F}(\gamma) \mathrm{d} \pi(\gamma)\right|=0
$$

Since we can choose the sequence $k \mapsto \bar{m}(k)$ strictly increasing, we can consider the sequence $N \mapsto \pi^{N}$ such that $\pi^{N} \in \mathcal{P}^{N}\left(\Gamma_{T}\right), \pi^{N}:=\bar{\pi}^{k}$ when $\bar{m}(k) \leq N<\bar{m}(k+1) ; \pi^{N}$ narrowly converges to $\pi$ as $N \rightarrow+\infty$ and

$$
\lim _{N \rightarrow+\infty} \int_{\Gamma_{T}} \mathfrak{F}(\gamma) \mathrm{d} \pi^{N}(\gamma)=\int_{\Gamma_{T}} \mathfrak{F}(\gamma) \mathrm{d} \pi(\gamma)
$$

Since all the components of $\mathfrak{F}$ are nonnegative and lower semicontinuous maps, by a combination of [4, Proposition 1.62 (a)] and [4. Proposition 1.80] we have that (6.34) yields in particular that the measures

$$
\sigma_{1}^{N}:=\mathcal{F} \pi+\mathcal{F} \pi^{N}, \quad \sigma_{2}^{N}:=\mathcal{G} \pi+\mathcal{G} \pi^{N}, \quad \sigma_{3}^{N}:=\mathcal{H} \pi+\mathcal{H} \pi^{N}, \quad \sigma_{4}^{N}:=\mathcal{L} \pi+\mathcal{L} \pi^{N}
$$

weakly converge to $\sigma_{1}:=2 \mathcal{F} \pi, \sigma_{2}:=2 \mathcal{G} \pi, \sigma_{3}:=2 \mathcal{H} \pi$ and $\sigma_{4}:=2 \mathcal{L} \pi$ respectively. In particular they are uniformly tight, so that for every $\varepsilon>0$ there exists $\bar{N}(\varepsilon) \in \mathbb{N}$ and a compact set $B_{\varepsilon}$ and such that

$$
B_{\varepsilon} \subset A_{N}, \quad\left(\pi+\pi^{N}\right)\left(\Gamma_{T} \backslash B_{\varepsilon}\right)+\int_{\Gamma_{T} \backslash B_{\varepsilon}}(\mathcal{F}+\mathcal{L}+\mathcal{H}+\mathcal{G}) \mathrm{d}\left(\pi+\pi^{N}\right) \leq \varepsilon \quad \text { for every } N \geq \bar{N}(\varepsilon) .
$$

STEP 3: (Definition of $\left(\boldsymbol{x}^{N}, \boldsymbol{u}^{N}\right)$ and convergence.) We define $\mu_{t}^{N}:=\left(e_{t}\right)_{\sharp} \pi^{N} \in \mathcal{P}^{N}\left(\mathbb{R}^{d}\right)$ and we denote by $\boldsymbol{x}^{N}$ a corresponding curve such that $\mu\left[\boldsymbol{x}^{N}\right]=\mu^{N}$. We define

$$
\begin{aligned}
\boldsymbol{f}^{N}(t, x) & :=\boldsymbol{F}^{N}\left(x, \boldsymbol{x}^{N}(t)\right)=\boldsymbol{F}\left(x, \mu_{t}^{N}\right), \quad \boldsymbol{v}^{N}(t, x):=\boldsymbol{v}(t, x)+\boldsymbol{f}(t, x)-\boldsymbol{f}^{N}(t, x) \\
u_{i}^{N}(t) & :=\boldsymbol{v}^{N}\left(t, x_{i}^{N}(t)\right)
\end{aligned}
$$

and $\boldsymbol{u}^{N}=\left(u_{1}^{N}, \ldots, u_{N}^{N}\right)$. Notice that

$$
\boldsymbol{f}(t, x)-\boldsymbol{f}^{N}(t, x) \in U \text { and } \boldsymbol{u}^{N} \in U^{N} \text { thanks to the compatibility condition (3.7). }
$$

We have that $\boldsymbol{\nu}^{N}:=\boldsymbol{\nu}\left[\boldsymbol{x}^{N}, \boldsymbol{u}^{N}\right]=\boldsymbol{v}^{N} \mu^{N}$. Since each component $x_{i}^{N}$ of $\boldsymbol{x}^{N}$ belongs to $A$, then the sequence $\left(\boldsymbol{x}^{N}, \boldsymbol{u}^{N}\right)$ belongs to $\mathscr{A}^{N}$, so that $\left(\mu^{N}, \boldsymbol{\nu}^{N}\right) \in \mathscr{A}$. Using the same computation of the proof of Proposition 5.3, taking into account that $\mu^{N}$ satisfies

$$
\partial_{t} \mu_{t}^{N}+\nabla \cdot\left((\boldsymbol{f}(t, \cdot)+\boldsymbol{v}(t, \cdot)) \mu_{t}^{N}\right)=0
$$

with (recall (6.23) and (6.35)

$$
f_{0}^{T} \int_{\mathbb{R}^{d}} \psi(\boldsymbol{v}(t, x)) \mathrm{d} \mu_{t}^{N}(x) \mathrm{d} t=\int_{\Gamma_{T}} \mathcal{F}(\gamma) \mathrm{d} \pi^{N}(\gamma) \leq 1+\mathcal{E}(\mu \mid \boldsymbol{\nu})
$$

for $N$ sufficiently big, we obtain by (6.20), (5.12) and (5.5) that

$$
\sup _{N \in \mathbb{N}} \sup _{t \in[0, T]} \int_{\mathbb{R}^{d}}|x| \mathrm{d} \mu_{t}^{N}(x)<+\infty, \quad \sup _{N \in \mathbb{N}} \sup _{t \in[0, T]} \int_{\mathbb{R}^{d}} \phi(|x|) \mathrm{d} \mu_{t}^{N}(x)<+\infty,
$$


which implies the uniform convergence $\mu^{N} \rightarrow \mu$ in $C\left([0, T] ; \mathcal{P}_{1}\left(\mathbb{R}^{d}\right)\right)$ and the uniform estimate

$$
\left|\boldsymbol{f}^{N}(t, x)\right| \leq M^{\prime}(1+|x|) \quad \text { for every } t \in[0, T], x \in \mathbb{R}^{d}, N \in \mathbb{N},
$$

for a suitable constant $M^{\prime}>0$. By a direct computation, using the assumption (3.8), we obtain that $\boldsymbol{\nu}^{N} \rightarrow^{*} \boldsymbol{\nu}$ in $\mathcal{M}\left([0, T] \times \mathbb{R}^{d} ; U\right)$.

STEP 4: (Definition and convergence of $\mathcal{F}^{N}$.) We define $\mathcal{F}^{N}: A \rightarrow[0,+\infty)$ by

$$
\mathcal{F}^{N}(\gamma):=f_{0}^{T} \psi\left(\boldsymbol{v}^{N}(t, \gamma(t))\right) \mathrm{d} t
$$

Here we show that the sequence $\mathcal{F}^{N}$ converges to $\mathcal{F}$ uniformly on every compact set $\Lambda \subset A_{h}$ for some $h \in \mathbb{N}$. To do it, we fix $\Lambda \subset A_{h}$ and we prove that for any $\gamma \in \Lambda$ and every sequence $\left(\gamma_{N}\right)_{N \in \mathbb{N}} \subset \Lambda$ such that $\sup _{t \in[0, T]}\left|\gamma_{N}(t)-\gamma(t)\right| \rightarrow 0$, we have $\mathcal{F}^{N}\left(\gamma_{N}\right) \rightarrow \mathcal{F}(\gamma)$ as $N \rightarrow+\infty$.

By the assumption (3.8) we have that

$$
\lim _{N \rightarrow+\infty}\left|\boldsymbol{f}\left(t, \gamma_{N}(t)\right)-\boldsymbol{f}^{N}\left(t, \gamma_{N}(t)\right)\right|=0, \quad \forall t \in[0, T] .
$$

Since $\mathcal{H}$ is continuous in $A_{h}$ it holds

$$
\lim _{N \rightarrow+\infty} \int_{0}^{T} \phi\left(\left|\boldsymbol{v}\left(t, \gamma_{N}(t)\right)\right|\right) \mathrm{d} t=\int_{0}^{T} \phi(|\boldsymbol{v}(t, \gamma(t))|) \mathrm{d} t .
$$

Since $\phi$ is strictly convex and superlinear, by Visintin's Theorem [62, Thm. 3] $\boldsymbol{v}\left(\cdot, \gamma_{N}(\cdot)\right)$ strongly converges in $L^{1}(0, T)$ to $\boldsymbol{v}(\cdot, \gamma(\cdot))$. Then, using also the continuity of $\psi$, along a subsequence (still denoted by $\left.\gamma_{N}\right)$ we have

$$
\lim _{N \rightarrow+\infty} \psi\left(\boldsymbol{v}\left(t, \gamma_{N}(t)\right)+\boldsymbol{f}\left(t, \gamma_{N}(t)\right)-\boldsymbol{f}^{N}\left(t, \gamma_{N}(t)\right)=\psi(\boldsymbol{v}(t, \gamma(t))), \quad \text { for a.e. } t \in[0, T] .\right.
$$

Since by (5.5) and (6.41) we have

$$
\left|\boldsymbol{f}\left(t, \gamma_{N}(t)\right)-\boldsymbol{f}^{N}\left(t, \gamma_{N}(t)\right)\right| \leq\left(M+M^{\prime}\right)\left(1+\left|\gamma_{N}(t)\right|\right)
$$

then, using the doubling property and the uniform convergence of $\gamma_{N}$, we can find a constant $C$ such that

$$
\psi\left(\boldsymbol{v}\left(t, \gamma_{N}(t)\right)+\boldsymbol{f}\left(t, \gamma_{N}(t)\right)-\boldsymbol{f}^{N}\left(t, \gamma_{N}(t)\right)\right) \leq C\left(1+\phi\left(\left|\boldsymbol{v}\left(t, \gamma_{N}(t)\right)\right|\right)\right) .
$$

By (6.44) the generalized dominated convergence theorem (see for instance [35. Thm. 4, page 21]) shows that

$$
\lim _{N \rightarrow+\infty} \int_{0}^{T} \psi\left(\boldsymbol{v}^{N}\left(t, \gamma_{N}(t)\right) \mathrm{d} t=\int_{0}^{T} \psi(\boldsymbol{v}(t, \gamma(t))) \mathrm{d} t .\right.
$$

STEP 5: (Definition and convergence of $\mathcal{L}^{N}$.) We define $\mathcal{L}^{N}: A \rightarrow[0,+\infty)$ by

$$
\mathcal{L}^{N}(\gamma):=f_{0}^{T} L^{N}\left(\gamma(t), \boldsymbol{x}^{N}(t)\right) \mathrm{d} t
$$

Here we show that the sequence $\mathcal{L}^{N}$ converges to $\mathcal{L}$ uniformly on every compact set $\Lambda \subset A_{h}$ for some $h \in \mathbb{N}$. As in step 4 , we fix $\Lambda \subset A_{h}$ and we prove that for every sequence $\left(\gamma_{N}\right)_{N \in \mathbb{N}} \subset \Lambda$, with $\sup _{t \in[0, T]}\left|\gamma_{N}(t)-\gamma(t)\right| \rightarrow 0$, we have $\mathcal{L}^{N}\left(\gamma_{N}\right) \rightarrow \mathcal{L}(\gamma)$ as $N \rightarrow+\infty$. Indeed, by (3.1),

$$
\lim _{N \rightarrow+\infty} L^{N}\left(\gamma_{N}(t), \boldsymbol{x}^{N}(t)\right)=L\left(\gamma(t), \mu_{t}\right), \quad \forall t \in[0, T] .
$$

Since $\left(\gamma_{N}\right)_{N}$ is bounded and $\mu^{N} \rightarrow \mu$ in $C\left([0, T] ; \mathcal{P}_{1}\left(\mathbb{R}^{d}\right)\right)$, by (3.1) we obtain that

$$
\sup _{N \in \mathbb{N}} L^{N}\left(\gamma_{N}(t), \boldsymbol{x}^{N}(t)\right)<+\infty .
$$


By dominated convergence we conclude.

STEP 6: (Conclusion.) By the growth assumption (6.20) and (6.41) on $\boldsymbol{f}, \boldsymbol{f}^{N}$, the doubling property of $\phi$ and (2.10) we have

$$
\mathcal{F}^{N}(\gamma) \leq C(1+\mathcal{F}(\gamma)+\mathcal{G}(\gamma)) \quad \forall \gamma \in A, \quad \forall N \in \mathbb{N} .
$$

Moreover, by (3.1) and the uniform convergence of $\mu^{N}$ to $\mu$, there exists a constant $C$ such that

$$
\mathcal{L}^{N}(\gamma) \leq \mathcal{L}(\gamma)+C \quad \forall \gamma \in A, \quad \forall N \in \mathbb{N} .
$$

Fix $\varepsilon>0$ and let $B_{\varepsilon}$ and $\bar{N}(\varepsilon)$ such that (6.36) holds and

$$
\left|\int_{\Gamma_{T}} \mathcal{F}(\gamma) \mathrm{d} \pi^{N}(\gamma)-\int_{\Gamma_{T}} \mathcal{F}(\gamma) \mathrm{d} \pi(\gamma)\right|<\varepsilon
$$

By (6.51), 6.52) and (6.36) we have

$$
\int_{\Gamma_{T} \backslash B_{\varepsilon}} \mathcal{F}^{N}(\gamma) \mathrm{d}\left(\pi+\pi^{N}\right)(\gamma) \leq \varepsilon, \quad \int_{\Gamma_{T} \backslash B_{\varepsilon}} \mathcal{L}^{N}(\gamma) \mathrm{d}\left(\pi+\pi^{N}\right)(\gamma) \leq \varepsilon \quad \forall N \geq \bar{N}(\varepsilon) .
$$

Moreover, from the previous step, there exists $\tilde{N}(\varepsilon)$ such that

$$
\sup _{\gamma \in B_{\varepsilon}}\left|\mathcal{F}^{N}(\gamma)-\mathcal{F}(\gamma)\right| \leq \varepsilon, \quad \sup _{\gamma \in B_{\varepsilon}}\left|\mathcal{L}^{N}(\gamma)-\mathcal{L}(\gamma)\right| \leq \varepsilon \quad \forall N \geq \tilde{N}(\varepsilon)
$$

Hence

$$
\begin{aligned}
\left|\int_{\Gamma_{T}} \mathcal{F}^{N}(\gamma) \mathrm{d} \pi^{N}(\gamma)-\int_{\Gamma_{T}} \mathcal{F}(\gamma) \mathrm{d} \pi(\gamma)\right| & \leq\left|\int_{B_{\varepsilon}} \mathcal{F}^{N}(\gamma) \mathrm{d} \pi^{N}(\gamma)-\int_{B_{\varepsilon}} \mathcal{F}(\gamma) \mathrm{d} \pi(\gamma)\right| \\
& +\left|\int_{\Gamma_{T} \backslash B_{\varepsilon}} \mathcal{F}^{N}(\gamma) \mathrm{d} \pi^{N}(\gamma)-\int_{\Gamma_{T} \backslash B_{\varepsilon}} \mathcal{F}(\gamma) \mathrm{d} \pi(\gamma)\right| \\
& \leq \varepsilon+2 \varepsilon, \quad \forall N \geq \max \{\bar{N}(\varepsilon), \tilde{N}(\varepsilon)\},
\end{aligned}
$$

which shows that

$$
\lim _{N \rightarrow \infty} \int_{\Gamma_{T}} \mathcal{F}^{N}(\gamma) \mathrm{d} \pi^{N}(\gamma)=\int_{\Gamma_{T}} \mathcal{F}(\gamma) \mathrm{d} \pi(\gamma)
$$

Analogously we obtain

$$
\lim _{N \rightarrow \infty} \int_{\Gamma_{T}} \mathcal{L}^{N}(\gamma) \mathrm{d} \pi^{N}(\gamma)=\int_{\Gamma_{T}} \mathcal{L}(\gamma) \mathrm{d} \pi(\gamma)
$$

\subsection{Convergence of minima.}

\section{Proof of Theorem 3.1.}

(Equicontinuity) Let $N$ be fixed and $s \leq t$. From the constraint (3.9) we get

$$
\begin{aligned}
W_{1}\left(\mu_{s}^{N}, \mu_{t}^{N}\right) & \leq \frac{1}{N} \sum_{i=1}^{N}\left|x_{i}^{N}(s)-x_{i}^{N}(t)\right| \\
& \leq \frac{1}{N} \sum_{i=1}^{N} \int_{s}^{t}\left|\boldsymbol{F}^{N}\left(x_{i}(r), \boldsymbol{x}^{N}(r)\right)\right| \mathrm{d} r+\frac{1}{N} \sum_{i=1}^{N} \int_{s}^{t}\left|u_{i}^{N}(r)\right| \mathrm{d} r \\
& \leq \tilde{C}(t-s)+\int_{s}^{t} \frac{1}{N} \sum_{i=1}^{N}\left|u_{i}^{N}(r)\right| \mathrm{d} r
\end{aligned}
$$


where $\tilde{C}:=A+2 B\left(\sup _{N}\left|\boldsymbol{x}^{N}(0)\right|_{N}+A T+\sup _{N} \int_{0}^{T}\left|\boldsymbol{u}^{N}(r)\right|_{N} \mathrm{~d} r\right) e^{2 B T}$ (see the proof of 44.6) which is uniformly bounded, since $\mu_{0}^{n}$ is converging in $\mathcal{P}_{1}\left(\mathbb{R}^{d}\right)$ and $\mathcal{E}\left(\mu^{N}, \boldsymbol{\nu}^{N}\right)$ is uniformly bounded.

By Remark 2.4, we can select an admissible function $\theta$ satisfying (2.24) with $\mathcal{K}:=\left\{\mu_{0}\right\} \cup\left\{\mu_{0}^{N}\right.$ : $N \in \mathbb{N}\}$. The uniform bound on $\mathcal{E}\left(\mu^{N}, \nu^{N}\right)$ implies that

$$
\sup _{N} \int_{0}^{T} \frac{1}{N} \sum_{i=1}^{N} \theta\left(\left|u_{i}^{N}(r)\right|\right) \mathrm{d} r<+\infty,
$$

by the convexity and superlinearity of $\theta$ there exists a uniform modulus of continuity $\omega:[0,+\infty) \rightarrow$ $[0,+\infty)$ such that $\sup _{N} \int_{s}^{t} \frac{1}{N} \sum_{i=1}^{N}\left|u_{i}^{N}(r)\right| \mathrm{d} r \leq \omega(t-s)$.

Hence we have just shown the equicontinuity property

$$
W_{1}\left(\mu_{s}^{N}, \mu_{t}^{N}\right) \leq \omega(|t-s|)+\tilde{C}|t-s| \quad \forall t, s \in[0, T] .
$$

(Compactness) From Theorem 5.3 we have

$$
\sup _{N \in \mathbb{N}} \sup _{t \in[0, T]} \int_{\mathbb{R}^{d}} \theta(|x|) \mathrm{d} \mu_{t}^{n}(x)<+\infty .
$$

This implies that the family $\left(\mu^{N}\right)_{N \in \mathbb{N}} \subset \mathcal{P}_{1}\left(\mathbb{R}^{d}\right)$ is relatively compact, (see e.g. [5. Prop. 7.1.5]).

The application of Ascoli-Arzelà Theorem provides a limit curve $\mu \in C\left([0, T] ; \mathcal{P}_{1}\left(\mathbb{R}^{d}\right)\right)$ and a subsequence, still denoted by $\mu^{N}$, such that

$$
\sup _{t \in[0, T]} W_{1}\left(\mu\left[\boldsymbol{x}^{N}\right]_{t}, \mu_{t}\right) \rightarrow 0
$$

Concerning the control part we write $\boldsymbol{\nu}^{N}=\boldsymbol{v}^{N} \mu^{N}$. Since $\mathcal{E}^{N}\left(\boldsymbol{x}^{N}, \boldsymbol{u}^{N}\right)$ is uniformly bounded, we have

$$
\sup _{N \in \mathbb{N}} \int_{0}^{T} \int_{\mathbb{R}^{d}} \psi\left(\boldsymbol{v}^{N}(t, x)\right) \mathrm{d} \mu_{t}^{N}(x) \mathrm{d} t<+\infty .
$$

By the superlinearity of $\psi$ and the convergence (6.59), using the same argument of the proof of [5. Th. 5.4.4] we obtain that there exist $\boldsymbol{v}:[0, T] \times \mathbb{R}^{d} \rightarrow U$ and a subsequence (again denoted by $\boldsymbol{v}^{N}$ ) such that

$$
\int_{0}^{T} \int_{\mathbb{R}^{d}} \psi(\boldsymbol{v}(t, x)) \mathrm{d} \mu_{t}(x) \mathrm{d} t<+\infty
$$

and

$\lim _{N \rightarrow \infty} \int_{0}^{T} \int_{\mathbb{R}^{d}} \boldsymbol{\xi}(t, x) \cdot \boldsymbol{v}^{N}(t, x) \mathrm{d} \mu_{t}^{N}(x) \mathrm{d} t=\int_{0}^{T} \int_{\mathbb{R}^{d}} \boldsymbol{\xi}(t, x) \cdot \boldsymbol{v}(t, x) \mathrm{d} \mu_{t}(x) \mathrm{d} t, \quad \forall \boldsymbol{\xi} \in C_{c}^{\infty}\left([0, T] \times \mathbb{R}^{d} ; \mathbb{R}^{d}\right)$.

This proves the convergence of $\boldsymbol{\nu}^{N} \rightarrow \boldsymbol{\nu}:=\boldsymbol{v} \mu$ in $\mathcal{M}\left([0, T] \times \mathbb{R}^{d} ; U\right)$ and the fact that $(\mu, \boldsymbol{\nu})$ satisfy (3.13).

\section{Proof of Theorem 3.3}

The first two claims are standard consequence of the $\Gamma$-convergence result Theorem 3.2 and the coercivity property stated in Theorem 3.1. We thus consider the third claim.

Let us fix $\mu_{0} \in \mathcal{P}_{1}\left(\mathbb{R}^{d}\right)$ with compact support and $(\mu, \boldsymbol{\nu}) \in P\left(\mu_{0}\right)$. By Theorem 3.2 we can find a sequence of discrete solutions $\left(\hat{\boldsymbol{x}}^{N}, \hat{\boldsymbol{u}}^{N}\right)$ corresponding to initial data $\hat{\boldsymbol{x}}_{0}^{N}$ supported in $\operatorname{supp}\left(\mu_{0}\right)$ and measures $\left(\hat{\mu}^{N}, \hat{\boldsymbol{\nu}}^{N}\right)$ converging to $(\mu, \boldsymbol{\nu})$ such that (3.19) and (3.20) holds. Theorem 3.2 also yields $\lim _{N \rightarrow \infty} E^{N}\left(\hat{\boldsymbol{x}}_{0}^{N}\right)=E\left(\mu_{0}\right)$.

Let now $\left(\boldsymbol{x}_{0}^{N}\right)_{N \in \mathbb{N}}$ be any other sequence satisfying (3.21) with $\left(\boldsymbol{x}^{N}, \boldsymbol{u}^{N}\right) \in P\left(\boldsymbol{x}_{0}^{N}\right)$ and $\mu^{N}=$ $\mu\left[\boldsymbol{x}^{N}\right], \boldsymbol{\nu}^{N}=\boldsymbol{\nu}\left[\boldsymbol{x}^{n}, \boldsymbol{u}^{N}\right]$. Applying Lemma 2.5 we deduce that the associated measures $\mu_{0}^{N}$ satisfy

$$
\lim _{N \rightarrow \infty} \mathcal{C}_{\phi}\left(\hat{\mu}_{0}^{N}, \mu_{0}^{N}\right)=0 .
$$


Up to a permutation of the initial points $\left(\hat{x}_{0,1}^{N}, \hat{x}_{0,2}^{N}, \cdots, \hat{x}_{0, N}^{N}\right)$ (and of the corresponding solutions $\left.\left(\hat{\boldsymbol{x}}^{N}, \hat{\boldsymbol{u}}^{N}\right)\right)$ which however leaves $\hat{\mu}_{0}^{N}, \hat{\mu}^{N}, \hat{\boldsymbol{\nu}}^{N}$ invariant, we may assume by (2.1) that

$$
c_{N}=\mathcal{C}_{\phi}\left(\hat{\mu}_{0}^{N}, \mu_{0}^{N}\right)=\frac{1}{N} \sum_{i=1}^{N} \phi\left(\left|\hat{x}_{0, i}^{N}-x_{0, i}^{N}\right|\right) .
$$

For $0<\delta<T$ and $\boldsymbol{y}^{N, \delta}:=\delta^{-1}\left(\hat{\boldsymbol{x}}_{0}^{N}-\boldsymbol{x}_{0}^{N}\right)$ we can then define a new competitor by

$$
\begin{aligned}
& \boldsymbol{x}^{N, \delta}(t):= \begin{cases}(1-t / \delta) \boldsymbol{x}_{0}^{N}+t / \delta \hat{\boldsymbol{x}}_{0}^{N} & \text { if } t \in[0, \delta), \\
\hat{\boldsymbol{x}}^{N}(t-\delta) & \text { if } t \in[\delta, T],\end{cases} \\
& \boldsymbol{u}_{i}^{N, \delta}(t):= \begin{cases}\boldsymbol{y}^{N, \delta}-\boldsymbol{F}^{N}\left(x_{i}^{N, \delta}, \boldsymbol{x}^{N, \delta}(t)\right) & \text { if } t \in[0, \delta), \\
\hat{\boldsymbol{u}}^{N}(t-\delta) & \text { if } t \in[\delta, T] .\end{cases}
\end{aligned}
$$

It is easy to check that $\left(\boldsymbol{x}^{N, \delta}, \boldsymbol{u}^{N, \delta}\right) \in \mathscr{A}\left(\boldsymbol{x}_{0}^{N}\right)$ so that $E^{N}\left(\boldsymbol{x}_{0}^{N}\right) \leq \mathcal{E}^{N}\left(\boldsymbol{x}^{N, \delta}, \boldsymbol{u}^{N, \delta}\right)$. On the other hand

$$
\begin{aligned}
T \mathcal{E}^{N}\left(\boldsymbol{x}^{N, \delta}, \boldsymbol{u}^{N, \delta}\right) & \leq \frac{1}{N} \int_{0}^{\delta} \sum_{i=1}^{N} L^{N}\left(x_{i}^{N, \delta}(t), \boldsymbol{x}^{N, \delta}(t)\right) \mathrm{d} t+\frac{1}{N} \int_{0}^{\delta} \sum_{i=1}^{N} \psi\left(\boldsymbol{y}^{N, \delta}-\boldsymbol{F}^{N}\left(x_{i}^{N, \delta}, \boldsymbol{x}^{N, \delta}(t)\right)\right) \mathrm{d} t \\
& +T \mathcal{E}^{N}\left(\hat{\boldsymbol{x}}^{N}, \hat{\boldsymbol{u}}^{N}\right) .
\end{aligned}
$$

From the doubling property and the compactness of supports of $\left(\boldsymbol{x}_{0}^{N}\right)$, applying the same argument as in the proof of Theorem 3.2 we get

$$
\begin{aligned}
\psi\left(\boldsymbol{y}^{N, \delta}-\boldsymbol{F}^{N}\left(x_{i}^{N, \delta}, \boldsymbol{x}^{N, \delta}(t)\right)\right) & \leq C\left(1+\phi\left(\left|\hat{\boldsymbol{x}}_{0}^{N}-\boldsymbol{x}_{0}^{N}\right| / \delta\right)\right) \\
& \leq C \mathrm{e}^{K / \delta}\left(1+\phi\left(\left|\hat{\boldsymbol{x}}_{0}^{N}-\boldsymbol{x}_{0}^{N}\right|\right)\right) \quad 0<\delta<1 .
\end{aligned}
$$

Setting $\mu_{t}^{N, \delta}=\mu\left[\boldsymbol{x}^{N, \delta}(t)\right]$ we get,

$$
T\left(\mathcal{E}^{N}\left(\boldsymbol{x}^{N, \delta}, \boldsymbol{u}^{N, \delta}\right)-\mathcal{E}^{N}\left(\hat{\boldsymbol{x}}^{N}, \hat{\boldsymbol{u}}^{N}\right)\right) \leq C c_{N} \delta\left(1+\mathrm{e}^{K / \delta}\right)+\delta \sup _{t \in[0,1]} \int_{\mathbb{R}^{d}} L^{N}\left(x, \mu_{t}^{N, 1}\right) \mathrm{d} \mu_{t}^{N, 1} .
$$

If we choose $\delta=\delta(N):=-K\left(\log \left(c_{N}\right)\right)^{-1}$, since $\lim _{N \rightarrow \infty} \sup _{t \in[0,1]} W_{1}\left(\mu_{t}^{N, 1}, \mu_{0}\right)=0$, we see that the right hand side of (6.61) tends to 0 as $N \rightarrow \infty$, so that we eventually obtain

$$
\limsup _{N \rightarrow \infty} E^{N}\left(\boldsymbol{x}_{0}^{N}\right) \leq \limsup _{N \rightarrow \infty} \mathcal{E}^{N}\left(\boldsymbol{x}^{N, \delta}, \boldsymbol{u}^{N, \delta}\right) \leq \limsup _{N \rightarrow \infty} \mathcal{E}^{N}\left(\hat{\boldsymbol{x}}^{N}, \hat{\boldsymbol{u}}^{N}\right)=E\left(\mu_{0}\right) .
$$

\section{Acknowledgements}

We wish to thank Filippo Santambrogio for useful discussions concerning the third claim of Theorem 3.3

Massimo Fornasier acknowledges the financial support provided by the ERC-Starting Grant "High-Dimensional Sparse Optimal Control" (HDSPCONTR) and the DFG-Project FO 767/71 "Identification of Energies from the Observsation of Evolutions". Giuseppe Savaré acknowledges the financial support provided by Cariplo foundation and Regione Lombardia via project "Variational evolution problems and optimal transport". Carlo Orrieri acknowledges the financial support provided by PRIN 20155PAWZB "Large Scale Random Structures". 


\section{References}

[1] Giacomo Albi, Mattia Bongini, Emiliano Cristiani, and Dante Kalise. Invisible control of self-organizing agents leaving unknown environments. SIAM J. Appl. Math., 76(4):1683$1710,2016$.

[2] Giacomo Albi, Young-Pil Choi, Massimo Fornasier, and Dante Kalise. Mean field control hierarchy. Appl. Math. Optim., 76(1):93-135, 2017.

[3] Luigi Ambrosio and Gianluca Crippa. Continuity equations and ODE flows with non-smooth velocity. Proc. Roy. Soc. Edinburgh Sect. A, 144(6):1191-1244, 2014.

[4] Luigi Ambrosio, Nicola Fusco, and Diego Pallara. Functions of bounded variation and free discontinuity problems. Oxford Mathematical Monographs. The Clarendon Press, Oxford University Press, New York, 2000.

[5] Luigi Ambrosio, Nicola Gigli, and Giuseppe Savaré. Gradient flows in metric spaces and in the space of probability measures. Lectures in Mathematics ETH Zürich. Birkhäuser Verlag, Basel, second edition, 2008.

[6] Daniel Andersson and Boualem Djehiche. A maximum principle for SDEs of mean-field type. Appl. Math. Optim., 63(3):341-356, 2011.

[7] Michele Ballerini, Nicola Cabibbo, Raphael Candelier, Andrea Cavagna, Evaristo Cisbani, Irene Giardina, Vivien Lecomte, Alberto Orlandi, Giorgio Parisi, Andrea Procaccini, et al. Interaction ruling animal collective behavior depends on topological rather than metric distance: Evidence from a field study. Proceedings of the national academy of sciences, 105(4):12321237, 2008.

[8] Erhan Bayraktar, Andrea Cosso, and Huyên Pham. Randomized dynamic programming principle and Feynman-Kac representation for optimal control of McKean-Vlasov dynamics. Trans. Amer. Math. Soc., 370(3):2115-2160, 2018.

[9] Alain Bensoussan, Jens Frehse, and Phillip Yam. Mean field games and mean field type control theory. SpringerBriefs in Mathematics. Springer, New York, 2013.

[10] Mattia Bongini and Massimo Fornasier. Sparse stabilization of dynamical systems driven by attraction and avoidance forces. Netw. Heterog. Media, 9(1):1-31, 2014.

[11] Mattia Bongini and Massimo Fornasier. Sparse control of multiagent systems. In Active particles. Vol. 1. Advances in theory, models, and applications, Model. Simul. Sci. Eng. Technol., pages 173-228. Birkhäuser/Springer, Cham, 2017.

[12] Mattia Bongini, Massimo Fornasier, and Dante Kalise. (Un)conditional consensus emergence under perturbed and decentralized feedback controls. Discrete Contin. Dyn. Syst., 35(9):40714094, 2015.

[13] Mattia Bongini, Massimo Fornasier, Francesco Rossi, and Francesco Solombrino. Mean-field Pontryagin maximum principle. J. Optim. Theory Appl., 175(1):1-38, 2017.

[14] Rainer Buckdahn, Boualem Djehiche, and Juan Li. A general stochastic maximum principle for SDEs of mean-field type. Appl. Math. Optim., 64(2):197-216, 2011.

[15] Scott Camazine, Jean-Louis Deneubourg, Nigel R. Franks, James Sneyd, Guy Theraulaz, and Eric Bonabeau. Self-organization in biological systems. Princeton Studies in Complexity. Princeton University Press, Princeton, NJ, 2003. Reprint of the 2001 original.

[16] Marco Caponigro, Massimo Fornasier, Benedetto Piccoli, and Emmanuel Trélat. Sparse stabilization and optimal control of the Cucker-Smale model. Math. Control Relat. Fields, 3(4):447-466, 2013. 
[17] René Carmona, François Delarue, and Aimé Lachapelle. Control of McKean-Vlasov dynamics versus mean field games. Math. Financ. Econ., 7(2):131-166, 2013.

[18] José A. Carrillo, Young-Pil Choi, and Maxime Hauray. The derivation of swarming models: mean-field limit and Wasserstein distances. In Collective dynamics from bacteria to crowds, volume 553 of CISM Courses and Lect., pages 1-46. Springer, Vienna, 2014.

[19] José A. Carrillo, Young-Pil Choi, and Sergio P. Perez. A review on attractive-repulsive hydrodynamics for consensus in collective behavior. In Active particles. Vol. 1. Advances in theory, models, and applications, Model. Simul. Sci. Eng. Technol., pages 259-298. Birkhäuser/Springer, Cham, 2017.

[20] José A. Carrillo, Maria R. D’Orsogna, and Vladislav Panferov. Double milling in self-propelled swarms from kinetic theory. Kinet. Relat. Models, 2(2):363-378, 2009.

[21] José A. Carrillo, Massimo Fornasier, Giuseppe Toscani, and Francesco Vecil. Particle, kinetic, and hydrodynamic models of swarming. In Mathematical modeling of collective behavior in socio-economic and life sciences, Model. Simul. Sci. Eng. Technol., pages 297-336. Birkhäuser Boston, Inc., Boston, MA, 2010.

[22] Young-Pil Choi, Seung-Yeal Ha, and Zhuchun Li. Emergent dynamics of the Cucker-Smale flocking model and its variants. In Active particles. Vol. 1. Advances in theory, models, and applications, Model. Simul. Sci. Eng. Technol., pages 299-331. Birkhäuser/Springer, Cham, 2017.

[23] Yao-li Chuang, Maria R. D'Orsogna, Daniel Marthaler, Andrea L. Bertozzi, and Lincoln S. Chayes. State transitions and the continuum limit for a 2D interacting, self-propelled particle system. Phys. D, 232(1):33-47, 2007.

[24] Yao-Li Chuang, Yuan R. Huang, Maria R. D’Orsogna, and Andrea L. Bertozzi. Multi-vehicle flocking: scalability of cooperative control algorithms using pairwise potentials. In Robotics and Automation, 2007 IEEE International Conference on, pages 2292-2299. IEEE, 2007.

[25] Iain D. Couzin and Nigel R. Franks. Self-organized lane formation and optimized traffic flow in army ants. Proceedings of the Royal Society of London B: Biological Sciences, 270(1511):139146, 2003.

[26] Iain D. Couzin, Jens Krause, Nigel R. Franks, and Simon A. Levin. Effective leadership and decision-making in animal groups on the move. Nature, 433(7025):513, 2005.

[27] Emiliano Cristiani, Benedetto Piccoli, and Andrea Tosin. Modeling self-organization in pedestrians and animal groups from macroscopic and microscopic viewpoints. In Mathematical modeling of collective behavior in socio-economic and life sciences, Model. Simul. Sci. Eng. Technol., pages 337-364. Birkhäuser Boston, Inc., Boston, MA, 2010.

[28] Emiliano Cristiani, Benedetto Piccoli, and Andrea Tosin. Multiscale modeling of granular flows with application to crowd dynamics. Multiscale Model. Simul., 9(1):155-182, 2011.

[29] Felipe Cucker and Jiu-Gang Dong. A general collision-avoiding flocking framework. IEEE Trans. Automat. Control, 56(5):1124-1129, 2011.

[30] Felipe Cucker and Ernesto Mordecki. Flocking in noisy environments. J. Math. Pures Appl. (9), 89(3):278-296, 2008.

[31] Felipe Cucker and Steve Smale. Emergent behavior in flocks. IEEE Trans. Automat. Control, $52(5): 852-862,2007$.

[32] Felipe Cucker and Steve Smale. On the mathematics of emergence. Jpn. J. Math., 2(1):197227, 2007. 
[33] Felipe Cucker, Steve Smale, and Ding-Xuan Zhou. Modeling language evolution. Found. Comput. Math., 4(3):315-343, 2004.

[34] Gianni Dal Maso. An introduction to $\Gamma$-convergence, volume 8 of Progress in Nonlinear Differential Equations and their Applications. Birkhäuser Boston, Inc., Boston, MA, 1993.

[35] Lawrence C. Evans and Ronald F. Gariepy. Measure theory and fine properties of functions. Textbooks in Mathematics. CRC Press, Boca Raton, FL, revised edition, 2015.

[36] Wendell H. Fleming. Generalized solutions in optimal stochastic control. pages 147-165. Lecture Notes in Pure and Appl. Math., 30, 1977.

[37] John J. Florentin. Optimal control of continuous time, Markov, stochastic systems. J. Electronics Control (1), 10:473-488, 1961.

[38] Massimo Fornasier, Benedetto Piccoli, and Francesco Rossi. Mean-field sparse optimal control. Philos. Trans. R. Soc. Lond. Ser. A Math. Phys. Eng. Sci., 372(2028):20130400, 21, 2014.

[39] Massimo Fornasier and Francesco Solombrino. Mean-field optimal control. ESAIM Control Optim. Calc. Var., 20(4):1123-1152, 2014.

[40] Guillaume Grégoire and Hugues Chaté. Onset of collective and cohesive motion. Physical review letters, 92(2):025702, 2004.

[41] Ali Jadbabaie, Jie Lin, and A. Stephen Morse. Correction to: "Coordination of groups of mobile autonomous agents using nearest neighbor rules" [IEEE Trans. Automat. Control 48 (2003), no. 6, 988-1001; MR 1986266]. IEEE Trans. Automat. Control, 48(9):1675, 2003.

[42] Jinyun Ke, James W. Minett, Ching-Pong Au, and William S.-Y. Wang. Self-organization and selection in the emergence of vocabulary. Complexity, 7(3):41-54, 2002.

[43] Evelyn F. Keller and Lee A. Segel. Initiation of slime mold aggregation viewed as an instability. Journal of Theoretical Biology, 26(3):399-415, 1970.

[44] Arthur L. Koch and David White. The social lifestyle of myxobacteria. Bioessays, 20(12):1030-1038, 1998.

[45] Harold J. Kushner. Optimal stochastic control. IRE Transactions on Automatic Control, $7(5): 120-122,1962$.

[46] Daniel Lacker. Limit theory for controlled McKean-Vlasov dynamics. SIAM J. Control Optim., 55(3):1641-1672, 2017.

[47] Mathieu Laurière and Olivier Pironneau. Dynamic programming for mean-field type control. C. R. Math. Acad. Sci. Paris, 352(9):707-713, 2014.

[48] Naomi Ehrich Leonard and Edward Fiorelli. Virtual leaders, artificial potentials and coordinated control of groups. In Decision and Control, 2001. Proceedings of the 40th IEEE Conference on, volume 3, pages 2968-2973. IEEE, 2001.

[49] Hiro-Sato Niwa. Self-organizing dynamic model of fish schooling. Journal of theoretical Biology, 171(2):123-136, 1994.

[50] Julia K. Parrish and Leah Edelstein-Keshet. Complexity, pattern, and evolutionary trade-offs in animal aggregation. Science, 284(5411):99-101, 1999.

[51] Julia K. Parrish, Steven V. Viscido, and Daniel Grunbaum. Self-organized fish schools: an examination of emergent properties. The biological bulletin, 202(3):296-305, 2002.

[52] Laura Perea, Pedro Elosegui, and Gerard Gómez. Extension of the cucker-smale control law to space flight formations. Journal of guidance, control, and dynamics, 32(2):527-537, 2009. 
[53] Benoît Perthame. Transport equations in biology. Frontiers in Mathematics. Birkhäuser Verlag, Basel, 2007.

[54] Huyên Pham and Xiaoli Wei. Bellman equation and viscosity solutions for mean-field stochastic control problem. to appear on ESAIM Control Optim. Calc. Var., 2018.

[55] William L. Romey. Individual differences make a difference in the trajectories of simulated schools of fish. Ecological Modelling, 92(1):65-77, 1996.

[56] Riccarda Rossi and Giuseppe Savaré. Tightness, integral equicontinuity and compactness for evolution problems in Banach spaces. Ann. Sc. Norm. Super. Pisa Cl. Sci. (5), 2(2):395-431, 2003.

[57] Martin B. Short, Maria R. D'Orsogna, Virginia B. Pasour, George E. Tita, P. Jeffrey Brantingham, Andrea L. Bertozzi, and Lincoln B. Chayes. A statistical model of criminal behavior. Math. Models Methods Appl. Sci., 18(suppl.):1249-1267, 2008.

[58] Ken Sugawara and Masaki Sano. Cooperative acceleration of task performance: Foraging behavior of interacting multi-robots system. Physica D: Nonlinear Phenomena, 100(3-4):343354, 1997.

[59] John Toner and Yuhai Tu. Long-range order in a two-dimensional dynamical xy model: how birds fly together. Physical review letters, 75(23):4326, 1995.

[60] Tamás Vicsek, András Czirók, Eshel Ben-Jacob, Inon Cohen, and Ofer Shochet. Novel type of phase transition in a system of self-driven particles. Phys. Rev. Lett., 75(6):1226-1229, 1995 .

[61] Tamás Vicsek and Anna Zafeiris. Collective motion. Physics Reports, 517(3-4):71-140, 2012.

[62] Augusto Visintin. Strong convergence results related to strict convexity. Comm. Partial Differential Equations, 9(5):439-466, 1984.

[63] Christian A. Yates, Radek Erban, Carlos Escudero, Iain D. Couzin, Jerome Buhl, Ioannis G. Kevrekidis, Philip K. Maini, and David J.T. Sumpter. Inherent noise can facilitate coherence in collective swarm motion. Proceedings of the National Academy of Sciences, 106(14):5464$5469,2009$. 